\title{
A fly model establishes distinct mechanisms for synthetic CRISPR/Cas9 sex distorters
}

\author{
Barbara Fasuloㄹ, Angela Meccariello ${ }^{1}$, Maya Morgan ${ }^{1}$, Carl Borufka $\oplus^{1}$, Philippos \\ Aris Papathanos $\circledast^{2 *}$, Nikolai Windbichler $\oplus^{1 *}$
}

1 Department of Life Sciences, Imperial College London, Sir Alexander Fleming Building, South Kensington Campus, London, United Kingdom, 2 Department of Entomology, Robert H. Smith Faculty of Agriculture,

Food and Environment, Hebrew University of Jerusalem, Rehovot, Israel

* p.papathanos@ mail.huji.ac.il (PAP); nikolai.windbichler@imperial.ac.uk (NW)

\section{G openaccess}

Citation: Fasulo B, Meccariello A, Morgan M, Borufka C, Papathanos PA, Windbichler N (2020) A fly model establishes distinct mechanisms for synthetic CRISPR/Cas9 sex distorters. PLoS Genet 16(3): e1008647. https://doi.org/10.1371/journal. pgen.1008647

Editor: Harmit S. Malik, Fred Hutchinson Cancer Research Center, UNITED STATES

Received: November 20, 2019

Accepted: February 3, 2020

Published: March 13, 2020

Peer Review History: PLOS recognizes the benefits of transparency in the peer review process; therefore, we enable the publication of all of the content of peer review and author responses alongside final, published articles. The editorial history of this article is available here: https://doi.org/10.1371/journal.pgen.1008647

Copyright: @ 2020 Fasulo et al. This is an open access article distributed under the terms of the Creative Commons Attribution License, which permits unrestricted use, distribution, and reproduction in any medium, provided the original author and source are credited.

Data Availability Statement: All relevant data are within the manuscript and its Supporting Information files.

\section{Abstract}

Synthetic sex distorters have recently been developed in the malaria mosquito, relying on endonucleases that target the $\mathrm{X}$-chromosome during spermatogenesis. Although inspired by naturally-occurring traits, it has remained unclear how they function and, given their potential for genetic control, how portable this strategy is across species. We established Drosophila models for two distinct mechanisms for CRISPR/Cas9 sex-ratio distortion- "Xshredding" and "X-poisoning"-and dissected their target-site requirements and repair dynamics. X-shredding resulted in sex distortion when Cas 9 endonuclease activity occurred during the meiotic stages of spermatogenesis but not when Cas 9 was expressed from the stem cell stages onwards. Our results suggest that $X$-shredding is counteracted by the NHEJ DNA repair pathway and can operate on a single repeat cluster of non-essential sequences, although the targeting of a number of such repeats had no effect on the sex ratio. X-poisoning by contrast, i.e. targeting putative haplolethal genes on the X chromosome, induced a high bias towards males (>92\%) when we directed Cas9 cleavage to the $\mathrm{X}$-linked ribosomal target gene RpS6. In the case of X-poisoning sex distortion was coupled to a loss in reproductive output, although a dominant-negative effect appeared to drive the mechanism of female lethality. These model systems will guide the study and the application of sex distorters to medically or agriculturally important insect target species.

\section{Author summary}

Harmful insect populations can be eliminated for a lack of females if they are made to produce mostly male offspring. There are genes that occur naturally that make males produce mostly sons and, although we don't know exactly how they work, this appears to coincide with damage to the X-chromosome during the production of sperm. Recently, we showed in a mosquito species that such sex-biasing genes could also be constructed artificially from first principles. To better understand if this works in other species too, we designed and built male-biasing genes of two types in the fruit fly and determined what is needed to for a shift towards males. We show how different ways of cutting the $\mathrm{X}$-chromosome DNA at different times with CRISPR, results in distinct outcomes and started to ask what 
Funding: This study was funded by the BBSRC under the research grant $\mathrm{BB} / \mathrm{P} 000843 / 1$ to NW. PAP was funded by the Italian Ministry Education, University and Research (MIUR-D.M. no. 79 04.02.2014), by the United States - Israel Binational Agricultural Research and Development Fund (Research Grant No. IS-5180-19) and by the Israel Science Foundation (Research Grant No. 2388/19). The funders had no role in study design, data collection and analysis, decision to publish, or preparation of the manuscript.

Competing interests: The authors have declared that no competing interests exist. cellular processes are involved in this. These models will help us to design such genes for the control of insect species that transmit disease or threaten crops.

\section{Introduction}

In a population of sexually reproducing organisms, a significant sex bias towards males is predicted to decrease the population's overall reproductive output. One factor is the fecundity of females, the sex with a lower rate of gamete production, which can exert a large influence on the size of a population. Forcing the sex-ratio towards males has thus long been regarded as a potential avenue for the genetic control of harmful insect pest or disease-vector populations. While the introduction of various forms of female-killing genetic traits could achieve this goal, more powerful strategies have also been theorized. Hamilton, for example, speculated that a population of a heterogametic species would become increasingly male-biased if, at each generation a mutant $\mathrm{Y}$ chromosome would favour its transmission over the $\mathrm{X}$-chromosome. The decline in female numbers would result in a reduced population size and eventually the collapse of the population [1]. Hamilton's thinking was inspired by records of distorter traits in Aedes aegypti and Culex pipiens that can produce extreme sex-ratios of $>90 \%$ males. Cytological observations during male meiosis showed broken $\mathrm{X}$-chromosomes suggesting a causal link with the male-bias phenotype $[2,3]$. These findings inspired the generation of artificial distorter traits [4] first by using His-Cys box homing endonucleases and subsequently RNAguided endonucleases. In the malaria vector Anopheles gambiae (A. gambiae) autosomal I-PpoI [5] or CRISPR/Cas9-bearing transgenes [6] were used to target sequences on the Xchromosome during male meiosis. Even if these autosomal X-shredders were self-limiting and thus less invasive and powerful compared to a driving Y chromosome, cage experiments with I-PpoI induced extreme male-biased sex-ratios and population collapse confirming the potential of this system for genetic control.

Mechanistically, the nature of the target locus for which successful X-shredding was demonstrated suggested a possible coalescence of different effects in the mosquito system. The target sites are situated within the A. gambiae $28 \mathrm{~S}$ rDNA cluster which simultaneously represents (i) a high-copy number repeat on the $\mathrm{X}$-chromosome, (ii) an essential gene for ribosome biogenesis and function, (iii) the nucleolar organizing region of the cell as well as (iv) a sequence adjacent to the centromere of the X-chromosome and (v) the predicted pseudo-autosomal region of the $\mathrm{X}$-chromosome mediating pairing with the $\mathrm{Y}$ chromosome during meiosis [7]. The possible conflation of effects induced by $\mathrm{X}$-shredding in the mosquito has been a source of uncertainty regarding the potential to transfer this paradigm to other important pest species. In particular, the X-shredding approach has not been clearly delineated from a related, recently-proposed strategy, based on the targeting of X-linked haploinsufficient genes (commonly ribosomal genes) with the intent to induce female lethality [8]. To delineate this mechanism, which is assumed not to alter gamete production and to come into effect only in the developing progeny, we refer to it as X-poisoning. X-poisoning would also generate a male biased progeny but would be expected to lead to a significant loss of reproductive output in the form of inviable female embryos. Reduced hatching is however a feature of the mosquito sex distortion system in some transgenic strains [9]. Although experiments suggested that carry over of endonuclease protein rather than insufficiency of the rDNA was responsible for this zygotic lethality [4], a contribution of altered target gene function to this effect could not be ruled out completely. 
Here, we have sought to disentangle and reconstitute these two strategies in Drosophila melanogaster by targeting with CRISPR/Cas9 both X-linked multicopy repeats and, in parallel, X-linked putative haplolethal genes essential for ribosome function. By doing so we have also sought to demonstrate that the $\mathrm{X}$-shredding mechanism is transferable, in principle, between species and between target genes and has potential applications beyond malaria vector control. We have also explored the efficiency of X-poisoning in biasing the sex-ratio as an alternative strategy for genetic control.

\section{Results}

\section{Assessing Cas9 and LbCpf1 activity of transgenic strains}

Due to the inadequacies of the Gal4/UAS system for directing transgene expression in later stages of spermatogenesis [10], we first generated multiple strains by random or site-directed transgenesis where the cas 9 or $c p f 1$ (cas $12 a$ ) coding sequences were placed under the direct transcriptional control of the $\beta t u b 85 D$ promoter. This regulatory element drives high expression of genes exclusively in the male germline, during the primary spermatocyte stage (at the onset of Meiosis I) and, as demonstrated in the mosquito [4, 5], this is the stage with the strongest evidence of $\mathrm{X}$-shredding activity. To evaluate CRISPR function, we first crossed $\beta t u b 85 D$ endonuclease bearing strains to flies containing a gRNA transgene targeting the X-linked, single copy white gene, used here as a phenotypic marker (gRNAs $w \_e x 3 \_2$ for Cas9 and $w \_e x 3 \_1$ for Cas12a/LbCpf1 were used respectively) [11]. The mutation of white results in individuals with white eyes due to a lack of pigment and we compared the activity of the different lines by counting the fraction of offspring with white eyes (S1 Fig, S1 Table). In the progeny of the transheterozygotes we found variability in mutation frequencies ranging from $25.5 \%$ with $\beta$ tub85D-cas $9^{20 D}$ (on the $\mathrm{X}$ chromosome) to $52.2 \%$ with $\beta$ tub85D-cas $9^{20 F}$ (on the third chromosome) likely due to differing expression levels of the endonuclease transgene. In addition, the level of activity of all the $\beta t u b 85 D$ driven lines was significantly lower when compared to cas 9 driven by the nanos (nos) promoter using the same gRNA ( $96.7 \%$ white eyes). LbCpf1 showed low levels of activity ( $0.6 \%$ white eyes) and for our further experiments we exclusively utilized Cas 9 . Unless specifically indicated, we used the $\beta t u b 85 D$-cas $9^{20 F}$ insertion on chromosome 3, which yielded the highest white mutation frequency of $52.2 \%$ for all experiments.

\section{Identification of $\mathrm{X}$-linked target sequences}

To identify X-linked sequences which could be targeted by CRISPR/Cas9 in the male germline we used two different approaches. First, using publicly available short read and long read Drosophila datasets, we employed the Redkmer pipeline [12] which we previously developed to identify putative X-linked repeat sequences that were not also present on other chromosomes from raw sequence data alone (S2 Fig). From the set of kmers of 25 nucleotides that passed our criteria including abundance, $\mathrm{X}$-linkage, and the lack of predicted off-target cleavage, we selected 8 target sequences for gRNA design located in multiple sites across the X-chromosome (S2 and S3 Figs). These target repeat sequences were predicted each to be confined to single chromosomal regions with the 5 most abundant sequences all located within annotated genes (esi-2.1, muc14a, hydra, CG33235 and CG15040). We made no assumption about the higherorder structure or the conservation of these putative repeat sequences between individuals, although all chosen gRNA repeat targets were also predicted to be multicopy sequences on the $\mathrm{X}$ when we searched the DmelR6.01 genome assembly (Fig 1B) and all mapped exclusively to scaffold X1 of a recent heterochromatin-enriched genome assembly [13] (S2 Table). The maximum predicted abundance of the top repeat sequences by the assemblies (155 hits) or redkmer (232 hits) suggests that the target repeats in Drosophila would be less repetitive than the target in Anopheles, i.e. the $28 \mathrm{~S}$ rDNA cluster with an array in excess of 500 repeats per genome. In a 
A
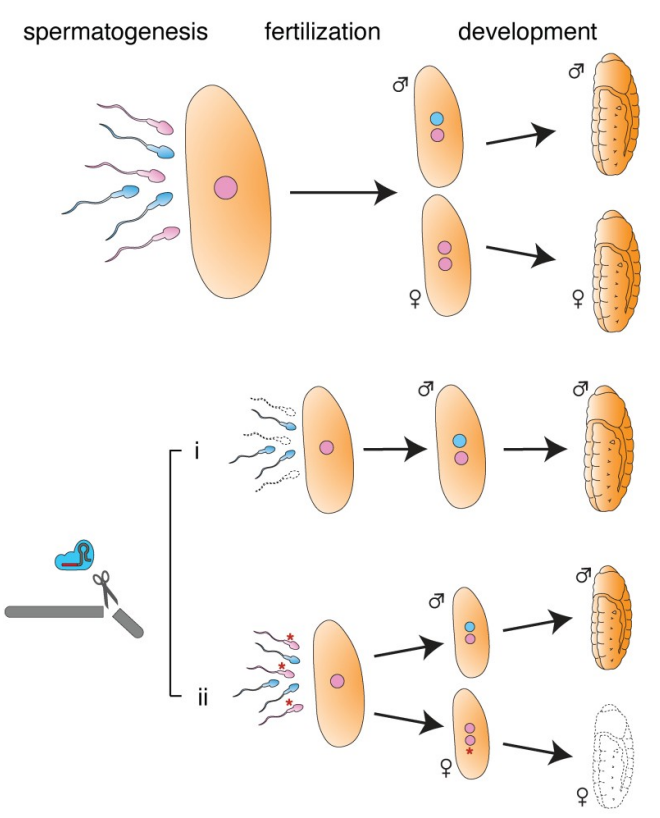

C

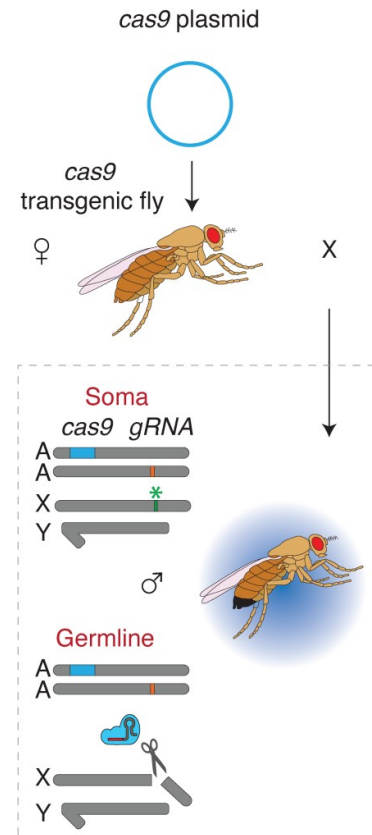

B

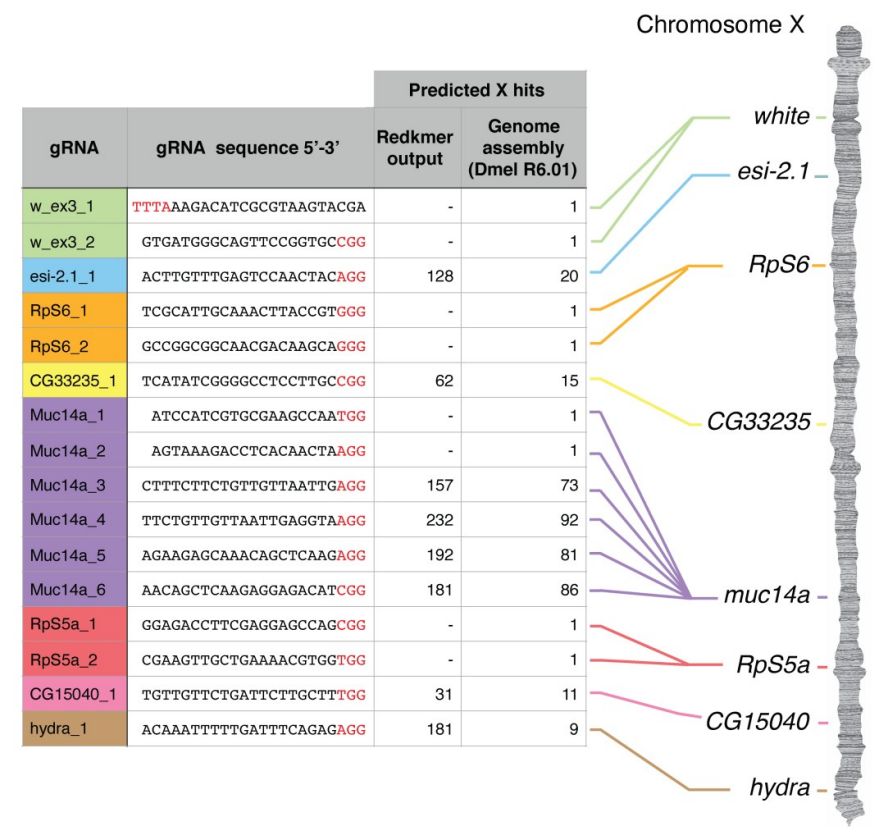

D

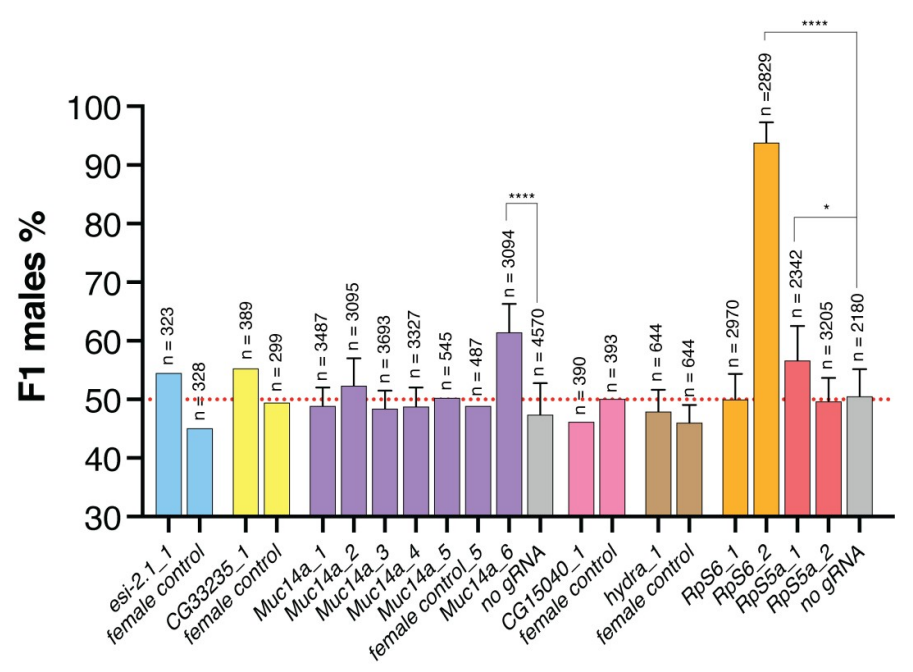

Fig 1. Development of sex ratio distorters in Drosophila (A) Model for pre-zygotic and post-zygotic effects on the reproductive sex-ratio. Compared to the unaltered process of development (top), an endonuclease targeting the X-chromosome during spermatogenesis (bottom) could either negatively affect the production or function of X-bearing sperm ( $\mathrm{i}$, prezygotic effect) or introduce genetic modifications that are detrimental to females inheriting such modified X-chromosomes during development (ii, postzygotic effect; e.g. the mutation of haploinsufficient genes). Pink: X-chromosome bearing gamete. Cyan: $\mathrm{Y}$ chromosome bearing gamete. The asterisk indicates gametes bearing modifications at the target site. (B) gRNA target sequences used in this study and their positions on the X-chromosome. The protospacer-adjacent motif (PAM) is indicated in red, and the X-chromosome hits predicted by Redkmer and the number of predicted perfect BLASTn hits to the X-chromosome in the Drosophila genome assembly (Dmel R6.01) are indicated. (C) Schematic of the experimental crosses. Females bearing the cas 9 transgene were crossed to males carrying the $g R N A$ transgene. Trans-heterozygote males were then crossed to wild-type females. Cas9 in concert with the gRNA cleaves the X-chromosome at the target site (green asterisk) in the germline and the effect is measured as the sex-ratio of the progeny. A: autosome; X: X-chromosome, Y; Y-chromosome. (D) Male sex-ratios in the offspring from crosses of $\beta$ tub85D-cas $9 / g R N A$ with wild-type $w$ females. Progeny of $\beta t u b 85 D$-cas $9 /+$ males crossed to wild type females (no gRNA) or from the reverse 
cross ( $\beta$ tub85D-cas9/gRNA females crossed to wild type males $=$ female control) served as a control. Crosses were set as pools of males and females or as multiple male single crosses in which case error bars indicate the mean \pm SD for a minimum of ten independent single crosses. For all crosses $n$ indicates the total number of individuals (males + females) in the $\mathrm{F} 1$ progeny counted. P-values ${ }^{*} \mathrm{p}<0.05,{ }^{* * * *} \mathrm{p}<0.0001$. All crossing data can be found in $\mathrm{S} 3$ Table.

https://doi.org/10.1371/journal.pgen.1008647.g001

second approach, we identified putative haploinsufficient genes [14], on the Drosophila Xchromosome and designed four gRNAs targeting conserved regions within the RpS5a [15] and RpS6 [16] ribosomal protein genes.

\section{Characterization of sex distorting gRNAs}

All gRNAs were cloned downstream of the RNA Polymerase III promoter of the Drosophila U6 snRNA gene that drives ubiquitous expression and we generated independent strains for all selected gRNAs. In addition, we also generated lines that combined either 2 different gRNAs using double dU6 promoters or 4 gRNAs expressed as a single transcriptional tRNAgRNA array $[17,18]$. The $\beta$ tub85D-cas 9 and each gRNA line were crossed to obtain trans-heterozygous males expressing both Cas 9 and the gRNA in the germline where activity is expected to occur. Next, these individuals were crossed to wild type females to determine the sex-ratio of their offspring (Fig 1C). As a control, we either used $\beta t u b 85 D$-cas 9 alone or the reciprocal cross that, due to the lack of $\beta t u b 85 D$ activity in females, was not expected to express Cas9. Fig 1D (S3 Table) summarizes the results of these experiments performed with a pool of individuals or by crossing single males to three females. We noted that while most gRNAs targeting Xlinked repeats did not substantially affect the sex-ratio, the Muc14a_6 gRNA induced a highly significant male-biased sex-ratio of $61.5 \%(\mathrm{p}<0.0001)$. This level of distortion was consistently observed in the follow-up experiments. Of those gRNAs targeting putative X-linked haplolethal genes, two gRNAs, RpS6_2 and Rps5a_1, yielded a significant excess of male progeny with frequencies of $93.8 \%(\mathrm{p}<0.0001)$ and $56.6 \%(\mathrm{p}=0.0112)$, respectively. We first focused our attention on the Muc14a_6 gRNA that we hypothesized to induce sex-ratio distortion by the X-shredding mechanism. The Muc14a_6 gRNA maps to the Mucin 14A (Muc14a, CG32580), an accessory-gland gene of unknown function that spans 52.6 kilobases and contains extended regions of tandemly repeated sequences (see also Fig 3A). The target repeats fall within the coding sequence of the gene with a predicted 271 nucleotides between individual Muc14a_6 gRNA target sites. We tested four gRNAs, each targeting different but interspersed repeat sequences in the $M u c 14 a$ gene and found that only one yielded significant sex distortion. Muc14a_6 gRNA produced an excess of male progeny even if its target was not predicted to be the one with the highest number of hits in the cluster (Fig 1B, S2 Table). To exclude the possibility that a loss of $M u c 14 a$ gene function is the cause of the sex-ratio distortion, we also designed two gRNAs, $M u c 14 a \_1$ and Muc14a_2, targeting putative non-repetitive regions in the Muc14a coding sequence. These experiments showed that the function of the Muc14a gene was not responsible for the male-biased sex-ratio observed in the progeny (Fig 1D).

We performed in-vitro Cas9 cleavage assays and our results suggested that all gRNAs targeting repeat sequences within the Muc14a locus were active in-vitro. For this purpose, we amplified and cloned a fragment of $1395 \mathrm{bp}$ of the Muc14a cluster that contained between 2 and 4 target sites for each of the four Muc14a gRNAs (Fig 2A) that served as a substrate for in-vitro digestions. We therefore concluded that the observed difference in their effects must be due to (i) different intrinsic or in-vivo gRNA performance, (ii) the particular nature or context of the target sequence or (iii) the specific repair outcomes triggered. We next tested combinations of gRNAs targeting the same or different X-linked repeat clusters to evaluate whether these could improve sex distortion rates (Fig 2B; S4 Table). While we observed a modest boost by 
A
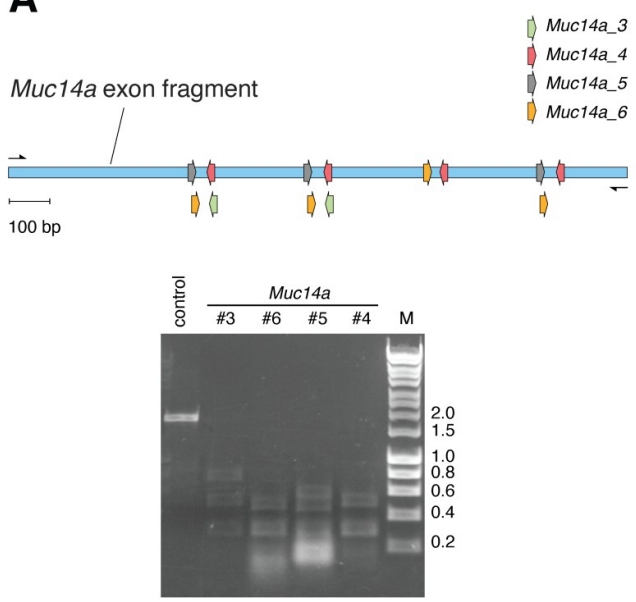

C

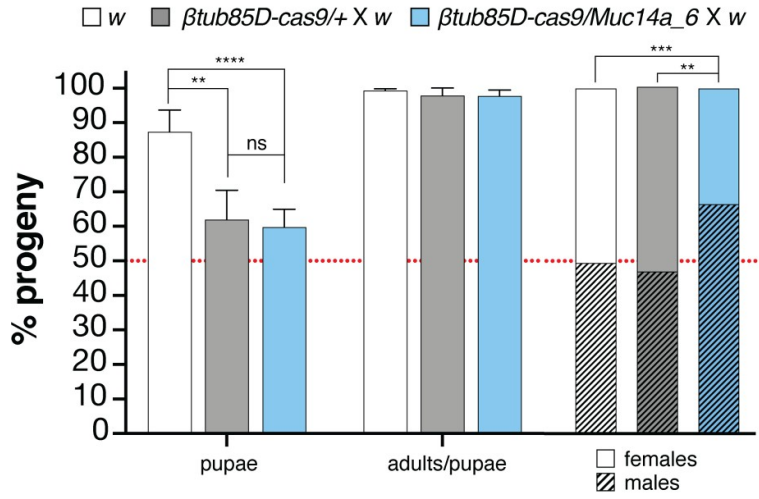

B

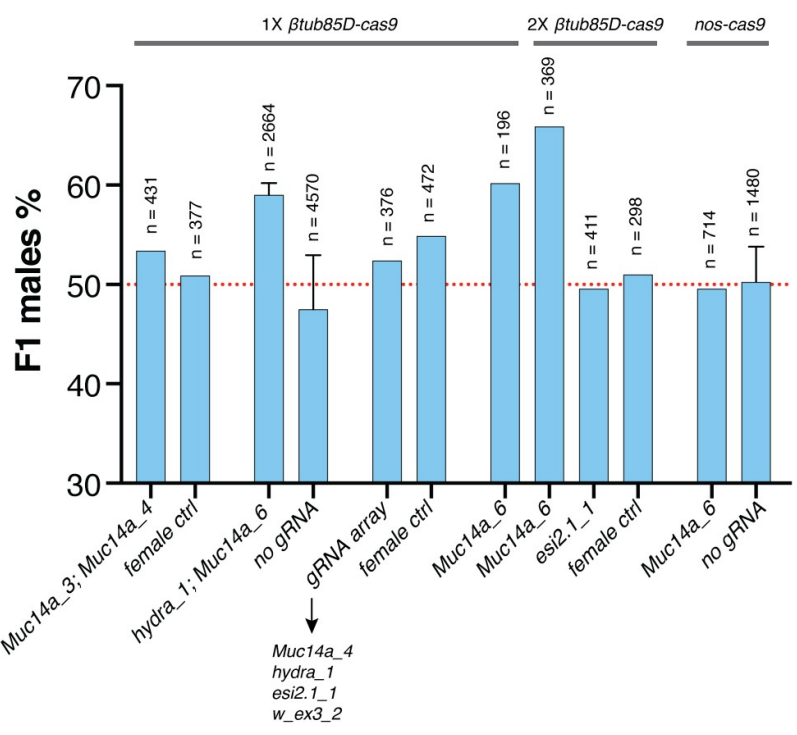

D

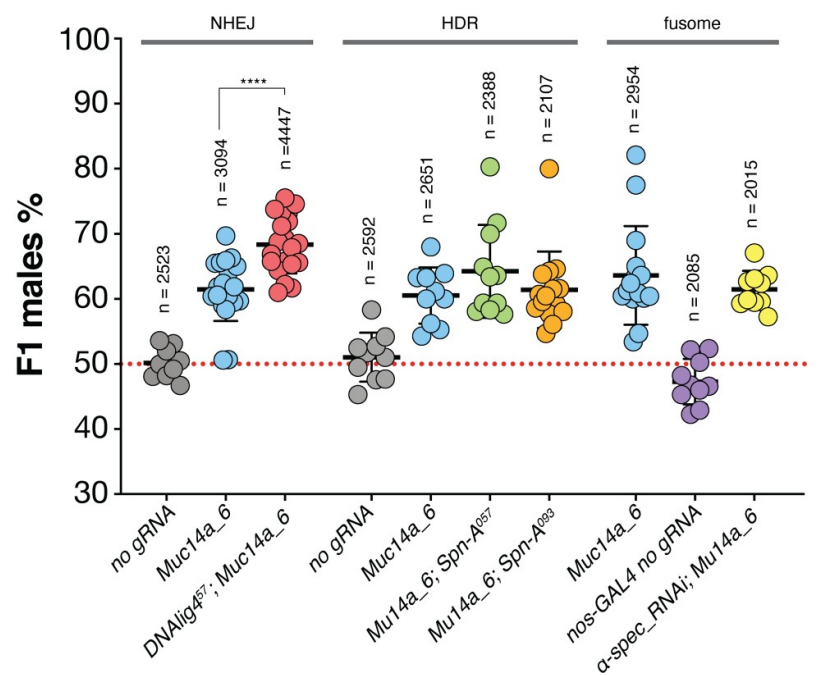

Fig 2. (A) Assay to detect CRISPR/Cas9-mediated cleavage in vitro. The region of the Muc14a gene that was amplified contains at least 2 binding sites for each of the gRNAs: Muc14a_3, Muc14a_4, Muc14a_5 and Muc14a_6 (top). The PCR amplified DNA fragment was used as a digestion target for Cas9/gRNA cleavage reactions in vitro (bottom). Reactions were run on a gel to detect cleavage. A control without gRNA was included. (B) Analysis of combinations of gRNAs and Cas9 sources for X-shredding. Average male frequencies in the F1 progeny are shown for each parental genotype with a single copy of $\beta$ tub85D-cas 9 transgene (1X), two copies of $\beta$ tub85D-cas 9 transgene ( $2 \mathrm{X}$ ) or one copy of nos-cas 9 (grey bars). All lines were crossed to wild type $w$ individuals. The reciprocal cross (female ctrl) or heterozygote $\beta$ tub85D-cas $9 /+$ or nos-cas $9 /+$ without gRNA (no gRNA) were used as control. The black arrow indicates gRNAs in the multiplex array and the red dotted line indicates an unbiased sex-ratio. Crosses were set as pools of males and females or as multiple male single crosses in which case error bars indicate the mean \pm SD for a minimum of ten independent single crosses. For all crosses $\mathrm{n}$ indicates the total number of individuals (males + females) in the F1 progeny counted. (C) Developmental survival analysis of the F1 progeny of Muc14a_6/קtub85D-cas 9 males crossed to $w$ females compared to $w$ and $\beta$ tub85D-cas $9 /+$ control males crossed to $w$ females. Left columns: embryos to pupae survival rate; central columns: pupae to adults survival rate and right columns: fraction of males and females in adults. Bars indicate means \pm SD for at least ten independent single crosses. Statistical significance was calculated with a $t$ test assuming unequal variance. ${ }^{* *} \mathrm{p}<0.01,{ }^{* * *} \mathrm{p}<0.001$ and ${ }^{* * * *} \mathrm{p}<0.0001$. (D) Influence of DNA repair and fusome integrity on $\mathrm{X}$ shredding. Male frequencies in the progeny of $D N A l i g 4^{57}$ (red), spn- $A^{057}$ (green) and spn- $A^{093}$ (orange) mutants and UAS_ $\alpha$-spectrinRNAi; nosGAL4 (yellow) knockdown mutants. The F1 sex-ratio of the progeny of males carrying Muc14a_6/3tub85D-cas 9 in the mutant backgrounds was compared with those of $\beta t u b 85 D$-cas9/+ (grey), Muc14a_6/ $\beta t u b 85 D$-cas9 (cyan) and nos-GAL4/+ (purple) control males. Each dot represents the percentage of $\mathrm{F} 1$ males from a cross between one male and three females. $\mathrm{n}$ is the number of individuals (males + females) in the F1 progeny. Bars show means $\pm \mathrm{SD}$ for at least ten independent single crosses. Statistical significance was calculated with a $t$ test assuming unequal variance. ${ }^{* * * *} \mathrm{p}<$ 0.0001. All crossing data in S3 Table. 
providing an additional Cas9 source, neither combination of gRNAs including the transcriptional array of four gRNAs resulted in a higher distortion than that of the single $M u c 14 a \_6$ gRNA alone. We also observed that the $w \_e x 3 \_2$ gRNA targeting white, when encoded as the most 3 ' of four consecutive gRNAs within the tRNA-gRNA array, showed a dramatically reduced level of activity (S1 Fig). In flies expressing both Muc14a_6 and hydra_1 gRNAs from separate loci we observed comparable distortion to that of $M u c 14 a \_6$ gRNA alone. Interestingly, we observed no sex-ratio distortion when nos-cas9 males, expressing the Muc14_6 gRNA, were crossed to wild type females. This result is in stark contrast with nos-cas 9 showing substantially higher levels of activity than $\beta t u b 85 D$-cas 9 when combined with the white gRNA (S1 Fig). As previously hypothesized [4], these findings support the idea that X-shredding is dependent on the timing of Cas9 activity, with expression in early spermatocytes also working well in the mosquito system. An analysis of Drosophila development in the progenies of $\beta t u b 85 D$-cas $9 /+$, $\beta t u b 85 D$-cas9/Muc14a_6 and wild type fathers confirmed that the Muc14a_6 gRNA induced no significant zygotic lethality-over any fitness effect of $\beta$ tub85D-cas 9 alone-an outcome expected if the loss of females is due to X-shredding acting pre-zygotically (Fig 2C; S5 Table).

\section{$\mathrm{X}$-shredding in backgrounds with impaired DNA repair activity or intracyst communication}

To better understand the cellular mechanisms that influence the outcome of X-shredding, i.e. loss of X-bearing gametes and a bias towards males in the progeny, we generated lines bearing mutations in DNA repair pathway components or core components of the fusome. The DNA Ligase IV gene (lig 4; CG12176) encodes an ATP-dependent DNA ligase involved in nonhomologous end joining (NHEJ) DNA repair; spindle-A (spn-A; CG7948) is a Drosophila homolog of the Rad51 gene required for double-strand break (DSB) repair by homologous recombination (HR) in both somatic and germ cells and $\alpha$-spectrin ( $\alpha$-spec; CG1977) encodes for a component of the fusome, an organelle that facilitates intracyst cell communications during spermatogenesis [19-21]. The fusome has also been implicated in the coordinated intracyst cell death response following DNA damage and mediating protein transport and diffusion between connected sperm cells, a process on which X-shredding in hemizygote individuals may rely on $[4,21]$. We found that the disruption of NHEJ repair by the mutant $\operatorname{lig} 4^{57}$ significantly increased the level of male-bias to above $68 \%$, whereas spn-A mutants and $\alpha$-spec dsRNAi did not affect the sex-ratio significantly (Fig 2D; S6 Table).

\section{Analysis of the Muc14a cluster by amplicon sequencing}

To gain a more accurate understanding of the DNA repair mechanisms acting during X-shredding we performed sequencing of the $M u c 14 a$ repeat cluster before and after it had undergone modification by Cas9. We crossed the Muc14a_6 gRNA strain to both, $\beta$ tub85D-cas 9 and noscas 9 flies to examine the outcomes of activity at different stages of spermatogenesis. However, since F1 females inherit modified X-chromosomes from their fathers and unmodified X-chromosomes from their mothers, it is difficult to assess the exact level of gene editing on paternal $\mathrm{X}$-chromosomes. To overcome this obstacle, we crossed $\beta t u b 85 D$-cas9/Muc14a_6 or nos-cas9/ Muc14a_6 male individuals to $\mathrm{X} \wedge \mathrm{X} / \mathrm{Y}$ females with attached-X-chromosomes [22]. By doing this, single modified X-chromosomes are passed from fathers to sons and can be analysed by amplicon sequencing (Fig $3 \mathrm{~A}$ ). In control males, $\sim 90 \%$ of repeats contained the full gRNA target site (Fig 3B, panel 1) although polymorphisms in the surrounding sequence indicated that the Muc14a cluster shows substantial heterogeneity. We found that, on average, X-chromosomes inherited from $\beta$ tub85D-cas $9 / M u c 14 a \_6$ fathers showed a $>40 \%$ reduction of cleavable gRNA target sites (Fig 3B, panel 1) but surprisingly this value was over $85 \%$, on average, in 
A

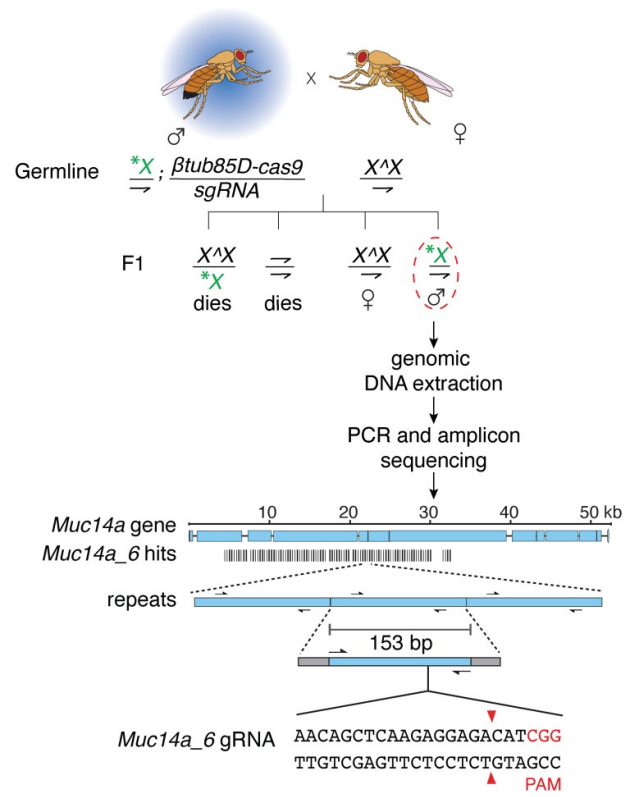

B
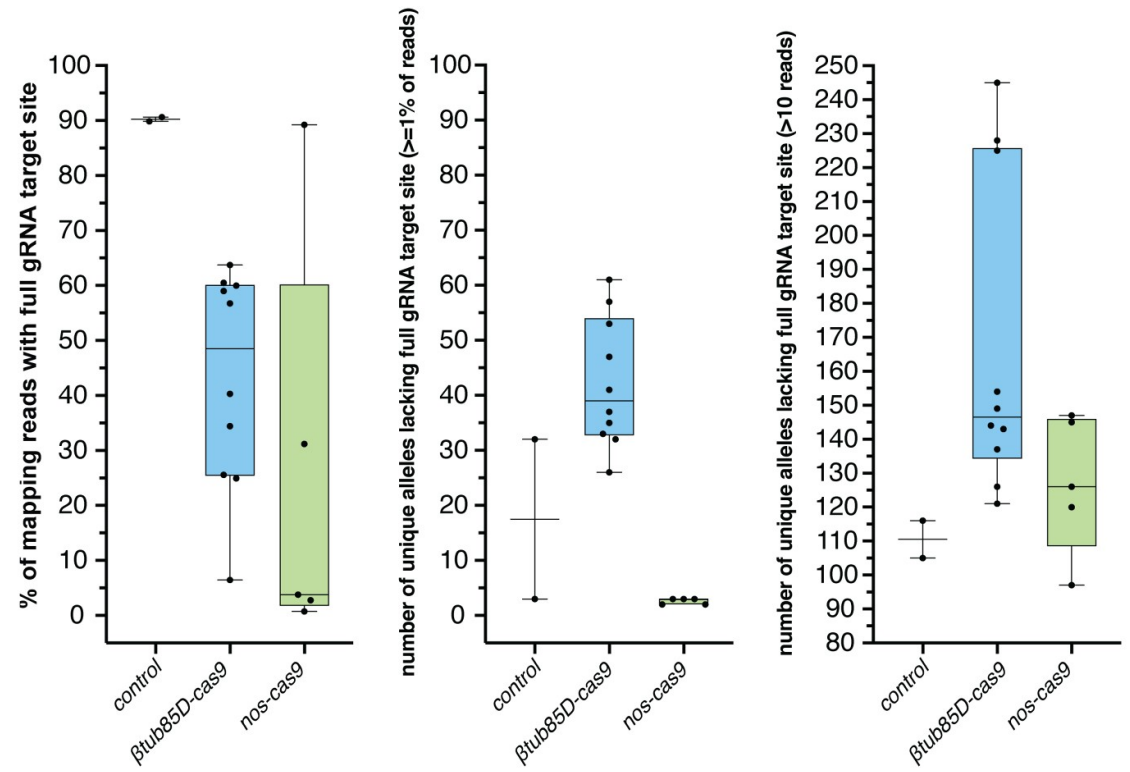

C

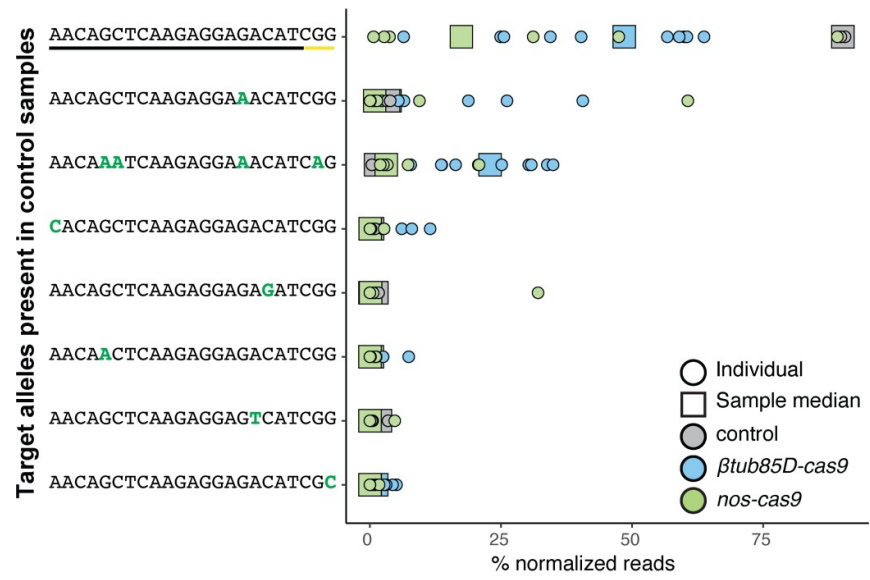

D

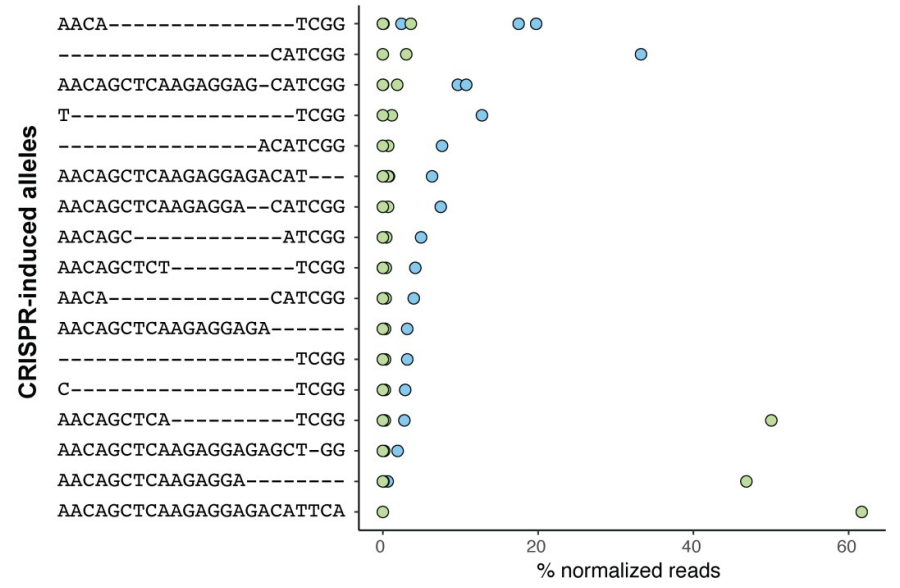

Fig 3. (A) Schematic of the genetic crosses to obtain shredded X-chromosomes from Muc14a_6/Btub85D-cas 9 or Muc14a_6/nos-cas 9 fathers for amplicon sequencing. Trans-heterozygous males for $\operatorname{cas} 9$ and the $g R N A$ were crossed to females with attached-X-chromosomes $\left(\mathrm{X}^{\wedge} \mathrm{X}\right)$. Offspring with supernumerary or lacking $\mathrm{X}$-chromosomes are inviable leaving $\mathrm{X} \wedge \mathrm{X} / \mathrm{Y}$ females and males carrying the $\mathrm{X}$-shredded chromosome (patroclinous inheritance) which we selected and analysed. Genomic DNA from single males was extracted and a $153 \mathrm{bp}$ DNA motif containing the Muc14a_6 gRNA target site was amplified with primers (thin arrows on repeats) containing Illumina Sequence adapters (grey boxes flanking the amplicon). As a comparison we used DNA from $\beta t u b 85 D$-cas $9 /+$ individuals lacking the gRNA. The shredded X-chromosome is indicated by a green asterisk. A dashed red circle indicates males selected for amplicon sequencing. Vertical black bars represent the number and location of the $M u c 14 a \_6$ gRNA repeats within the Muc14a gene. (B) Analysis of allele variation at the Muc14a_6 target site by amplicon sequencing. Indicated is the percentage of all mapped reads that contain the complete, unaltered Muc14a_6 target site (left panel) in the control and experimental males. The middle and right panel show the number of reads harbouring all other unique alleles that represented at least $1 \%$ of all reads or were represented by 10 or more reads, respectively. (C) Analysis of unique alleles (including the wild type target site) at the Muc14a_6 target site that pre-existed in both control samples. Indicated are the relative frequencies of these alleles in each control and experimental male (circles) including the median frequency of each allele in all Muc14a_6/Btub85D-cas9 or Muc14a_6/nos-cas9 males (squares). (D) Analysis of de-novo alleles at the Muc14a_6 target site not present in control males and putative CRISPR-induced alleles. In $\mathrm{C}$ and $\mathrm{D}$ we considered only alleles that represented $\geq 1 \%$ of normalized reads in at least one male sample.

https://doi.org/10.1371/journal.pgen.1008647.g003 
those X-chromosomes derived from nos-cas9/Muc14a_6 males, despite the fact that no sexratio distortion had been observed with this combination. X-chromosomes of $\beta t u b 85 D$-cas 9 / Muc14a_6 fathers had, on average a larger number of unique alleles (identified from mapping reads that lacked the gRNA at the target site) than the wild type. By contrast, the progeny of nos-cas9/Muc14a_6 showed a reduction of allele diversity, when considering alleles represented in $\geq 1 \%$ of mapping reads (Fig 3B, panel 2). A similar pattern was observed when we considered alleles with a read coverage above $10 \mathrm{X}$, where $\mathrm{X}$-chromosomes derived from Btub85D-cas9/Muc14a_6 males showed a more diverse repeat landscape compared to control and nos-cas9/Muc14a_6 males (Fig 3B, panel 3). We analysed in more detail the fate of preexisting alleles in the control males (Fig 3C) as well as novel alleles presumably generated by CRISPR/Cas9 activity (Fig 3D). While consensus allele frequency decreased in males of the experimental groups, we found a pre-existing allele predicted to be cleavage-resistant (AACAaATCAAGAGGAAACATCaG, with mutations in both the target site and the PAM), to increase in median frequency from $<1 \%$ to $\sim 25 \%$ in males from $\beta$ tub85D-cas $9 / M u c 14 a \_6$ but not from nos-cas9/Muc14a_6 fathers (Fig 3C, row 3). In contrast, novel CRISPR-induced alleles, likely generated pseudo-randomly, were commonly shared amongst a few males, although their mapping reads accounted for more than $40 \%$ of all reads in some males from nos-cas9/Muc14a_6 fathers (Fig 3D). On the sequence level, CRISPR-induced alleles consisted mainly of smaller deletions at or around the site of cleavage and all would be predicted to prevent further Cas9 cleavage. Together these findings suggest quite different dynamics of repair or repair mechanisms between the early acting nos-cas 9 and the meiotic $\beta t u b 85 D$-cas 9 .

\section{Targeting putative haplolethal $\mathrm{X}$-linked genes}

We next focussed our analysis on the set of gRNAs targeting putative haploinsufficient singlecopy genes. As we observed for gRNAs targeting the Muc14a repeats, no combination of gRNAs with $\beta$ tub85D-cas 9 was found to increase the sex-ratio relative to the RpS6_2 gRNA alone. Both gRNAs, RpS5a_1 and RpS6_2, when crossed to $\beta t u b 85 D$-cas 9 were individually capable of inducing male-bias sex-ratio distortion, but when combined within a gRNA array, we found a significantly lower level of distortion in the progeny than when we used RpS6_2 alone (Fig 4B; S7 Table). In contrast to X-shredding, X-poisoning is expected to act post-zygotically by inducing female lethality in the developing embryo likely as a result of an insufficient dose of the target-gene product. We examined Drosophila development in the progenies of $\beta t u b 85 D$-cas9/+, $\beta t u b 85 D$-cas9/RpS6_2 and wild type fathers and, in contrast to the Muc14a_6 gRNA, we did observe significant lethality at the embryonic and subsequent stages. This result would suggest a mechanism of lethality that operates during development and that the few survivor females in the progeny carried rescue mutations that restored the function of the ribosomal target genes, while preventing further CRISPR cleavage. To confirm this hypothesis, we performed amplicon sequencing of the RpS6_2 target sequence using 2 pools of the surviving females from $\beta t u b 85 D$-cas 9 fathers expressing the RpS6_2 or RpS6_2 and RpS6_1 gRNAs (Fig $4 \mathrm{C}$; S8 Table). Surprisingly, while we did observe possible rescue alleles, the majority of mutant alleles identified in surviving females are not expected to restore $R p S 6$ gene function because of the presence of translational frameshifts (Fig 4D). Although, one has to be cautious to infer loss of function from such experiments alone [23], these results imply that the mechanism of lethality following RpS6 cleavage and imperfect repair is dominant rather than dependent on an insufficient gene dose. Co-expression of Muc14_6 and Rps6_2 gRNAs, i.e. combining the two mechanism of distortion we have described, yielded the highest level of distortion we observed in this study (on average $95.8 \%$ males) although this was not significantly different from RpS6_2 alone. Finally, when we combined the Rps6_2 gRNAs with nos-cas 9 the result 
A

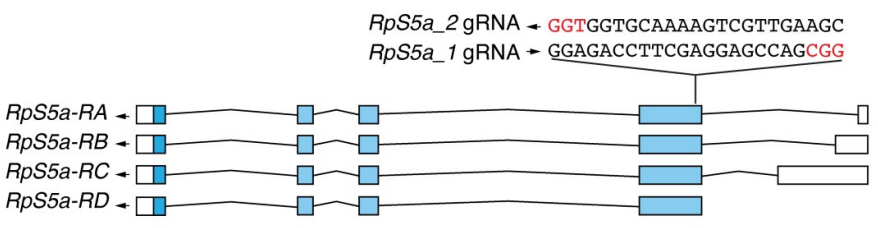

$R p S 6 \_1$ gRNA - TCGCATTGCAAACTTACCGTGGG

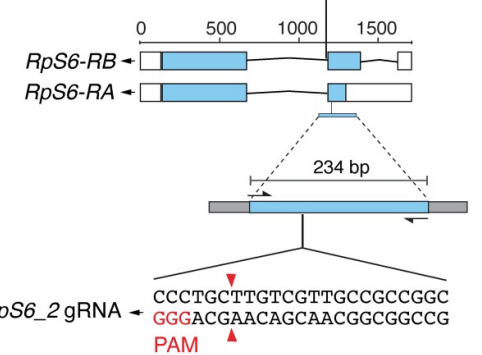

C

$\square w \quad \square$ Btub85D-cas920F/+ $\square$ Btub85D-cas920F/RpS6_2

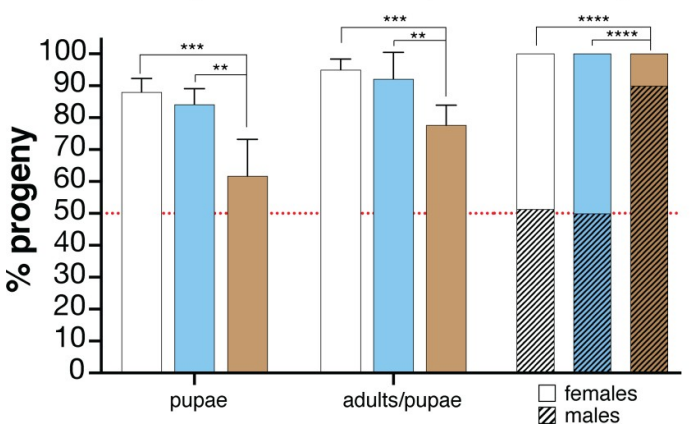

B

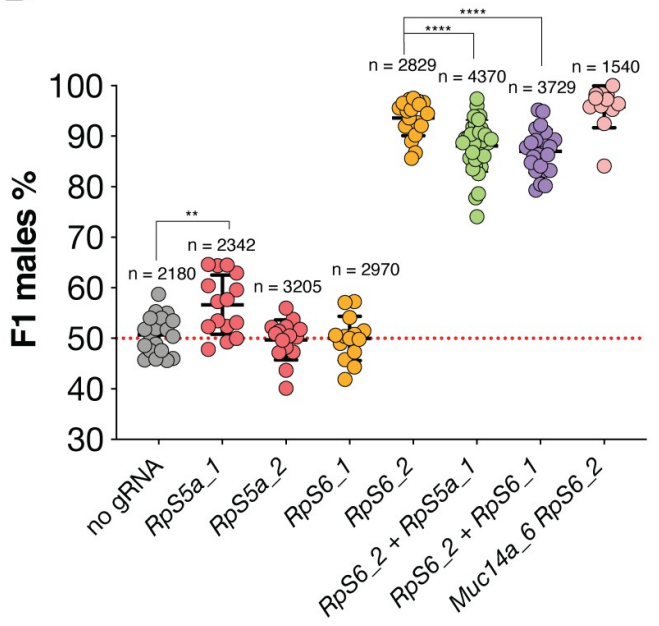

Fig 4. (A) gRNA target sites within presumptive haploinsufficient genes on the X-chromosome. Schematic representation of RpS5a and RpS6 gene organization. Both genes encode for a small ribosomal subunit protein (RpS). As illustrated in the figure, the RpS5a gRNAs, RpS5a_1 and _2, map in the fourth exon shared by all four transcripts of the gene and the RpS6 gRNAs, RpS6_1 and 2, map in the third exon of two transcripts in the corresponding gene. The figure shows the $234 \mathrm{bp}$ fragment (with Illumina Sequence adapters on both sides as grey boxes) surrounding the RpS6_2 gRNA target site that was used for amplicon sequencing. Blue boxes indicate coding sequences, white boxes indicate UTR regions and PAMs are indicated in red. (B) Efficiency of single gRNAs or combinations of gRNAs for X-poisoning. Shown is the frequency of males in the progeny from $\beta t u b 85 D$-cas 9 males combined with four $g R N A$ lines and crossed to wild type $w$ females. Individual gRNAs, RpS5a_1 or RpS5a_2(red), and RpS6_1 or RpS6_2 (orange) are compared to double gRNA arrays co-expressing RpS6_2 + RpS5a_1 (green) or RpS6_2 + RpS6_1 (purple) as well as a combination of both RpS6_2 and Muc14a_6 transgenes (pink). As a control, crosses from $\beta t u b 85 D$-cas $9 /+$ (no gRNA, grey) fathers are shown. Each dot represents the percentage of $\mathrm{F} 1$ males from a cross between one male and three females. $\mathrm{n}$ is the number of individuals (males + females) in the F1 progeny. Black bars show means \pm SD for at least ten independent single crosses. Statistical significance was calculated with a $t$ test assuming unequal variance. ${ }^{* *} \mathrm{p}<0.01,{ }^{* * *} \mathrm{p}<0.001$ and ${ }^{* * * *} \mathrm{p}<0.0001$. All crossing data can be found in S7 Table. (C) Developmental survival analysis of the F1 progeny of RpS6_2/Btub85D-cas9, $w$ or $\beta$ tub85D-cas9/+ males crossed to $w$ females. Left columns: embryos to pupae survival rate; central columns: pupae to adults survival rate and right columns: fraction of males and females in adults. Bars indicate means \pm SD for at least ten independent single crosses. Statistical significance was calculated with a $t$ test assuming unequal variance. ${ }^{* *} \mathrm{p}<0.01,{ }^{* * *} \mathrm{p}<0.001$ and ${ }^{* * * *} \mathrm{p}<0.0001$. All crossing data can be found in S8 Table. (D) CRISPR induced target site mutations within the RpS6 gene analysed by pooled amplicon sequencing of surviving female progeny. On top, the wild type DNA sequence spanning the RpS6_2 gRNA target site in the RpS6 ribosomal gene is shown with the gRNA binding position (underline), the cut site (red arrowhead), the PAM (bold nucleotides) as well as the encoded amino acids (blue). Target site variants identified in pools of F1 females from RpS6_2/Btub85D-cas 9 fathers or RpS6_2 RpS5a_1/Btub85D-cas9 fathers are shown below. Dashed lines correspond to nucleotide deletions, green coloured bases represent insertions. Predicted amino-acid substitutions $(>)$ are indicated to the right.

https://doi.org/10.1371/journal.pgen.1008647.g004

was complete sterility of the transheterozygous males (Supplementary Dataset 1). Since ribosomal gene function is required during gametogenesis, disrupting $R p S 6$ during the stem cell stages appears to be detrimental for sperm development. 


\section{Discussion}

To recreate in Drosophila a synthetic X-shredding mechanism pioneered in A. gambiae, we generated gRNAs targeting repeat sequences we identified on the X-chromosome. This demonstrates that $\mathrm{X}$-shredding is a transferable mechanism and not linked to the particular nature of the mosquito target, i.e. the ribosomal DNA gene cluster that was targeted in previous studies $[5,6]$. It also shows that CRISPR/Cas9 target sequences that are able to induce sex-ratio distortion can be identified bioinformatically. To search for such X-linked repeats, we employed the Redkmer pipeline using raw sequence data as the only input. This is crucial as many target species of medical or agricultural importance are likely to lack high-quality genome assemblies. Even when such assemblies exist, repeats sequences, a problematic class of sequences for assemblers, often remain poorly resolved. However, recent progress in telomere-to-telomere chromosome assemblies that incorporate large DNA repeat clusters may simplify this step in the future [24]. The level of sex distortion towards males we observed in the fly was not as extreme as observed in A. gambiae. Given that activity against the single-copy white was incomplete this suggests possible improvements by further optimizing the level of cas 9 expression in the germline of Drosophila. Using our best cas 9 strain, we found that achieving higher rates of distortion required us to interfere with mechanisms of DNA repair. This in turn indicates that targeting more repetitive sequences could be another avenue to enable a more dramatic bias towards males, though the Drosophila X-chromosome lacks such repeats. The smaller repeat unit size and hence smaller distance between individual cut sites (271 base pairs in the case of Muc14a_6 versus $\sim 9 \mathrm{~kb}$ in the A. gambiae rDNA target) could also have increased the chances of successful repair. In addition to the intrinsic activity of each gRNA, the sequence microenvironment rather than the number of repeats may also play a role in determining why certain gRNAs trigger gamete loss while others don't. The identification of X-chromosomes which are more suitable for $\mathrm{X}$-shredding in target species of medical or agricultural relevance is thus the next task. Our study provides lessons for the application of X-shredding to such species. A strong meiotic promoter yielding high levels of Cas9 should be combined with as few gRNAs as possible, ideally one, targeting a highly repetitive sequence, which does not have to be essential and can consist of a single repeat cluster on the X-chromosome.

Chromosome-wide, distributed repeats represent an alternative set of targets but the lack of such sequences in Drosophila precluded us from evaluating their use for X-shredding. The fly literature suggests that the $1.688 \mathrm{X}$-chromosome satellite involved in $\mathrm{X}$ dosage compensation [25] one of the most abundant repetitive sequences in Drosophila melanogaster, would represent such a target [26]. While the set of candidate targets did include a number of kmers that could be attributed to the 1.688 satellite they did not pass our selection criteria or were significantly less abundant than the targets we selected. This is likely due to the heterogeneity and stratification of 1.688 repeats in various chromosomal locations [27].

Although the use of larger arrays of gRNAs has been suggested to compensate for the lack of abundant X-linked repeats [6], our data suggests that targeting a highly repetitive sequence with a single gRNA may be a more viable route. Even if multiple gRNAs could be expressed efficiently and concomitantly, they could compete for access to Cas9 protein in the loading step thus reducing activity of each individual gRNA. The saturation of Cas9 by gRNAs has been observed previously [28].

Our data suggests that the high level of activity of nos-cas 9 with $M u c 14 a \_6$ gRNA during early spermatogenesis could cause the repeats to be subject to multiple cleavage-repair cycles which in turn may lead to the observed reduction in the complexity and presumably also the size of the repeat cluster (and no sex bias). By contrast, $\beta t u b 85 D$-cas9/Muc14a_6 activity, which acts later in meiosis, would encounter, following multiple mitotic divisions, more $\mathrm{X}$ - 
chromosomes to target in the larger population of primary spermatocytes on which it may be acting continuously and in parallel to generate novel alleles. This would result in a broader spectrum of mutations inherited by the progeny. Alternatively, the observed differences could also partly relate to the predominance of homologous over non-homologous DNA repair pathways acting with varying stringency during the early (including stem cells) and late stages of spermatogenesis, respectively [29] or an overall greater repair efficiency in pre-meiotic stages compared to later stages when $\beta$ tub85D-cas 9 is active. While nos-cas 9 would favour the loss of repeat units by recombination-based repair mechanism, $\beta$ tub85D-cas 9 would trigger NHEJ repair events leading to a more diverse allele pool. Indeed, we found little evidence of homologous repeat to repeat repair in $\beta t u b 85 D$-cas9/Muc14a_6 males. For instance, the pre-existing cleavage-resistant allele AACAaATCAAGAGGAAACATCaG is associated with a $6 \mathrm{bp}$ indel polymorphism located 53 nucleotides upstream of the gRNA target. We did not detect dissociation of this SNP even in samples in which the cleavage resistant allele rose to a frequency of $30 \%$ of the mapped read pool. Dissociation would have indicated that a double-strand break in a wild-type repeat unit had been repaired by gene conversion using the cleavage resistant allele as a template.

One caveat is the fact that the X-chromosomes from $\beta t u b 85 D$-cas9/Muc14a_6 males we analysed by amplicon sequencing managed to escape the germline in the form of $\mathrm{X}$-bearing gametes and gave rise to viable males. One might argue that they represent chromosomes with an overall lower level of modifications or modifications with repair outcomes compatible with transmission and male survival (which may in turn differ from the requirements for female survival). In contrast, the $\mathrm{X}$-chromosomes unable to form viable gametes, i.e. those that we could not analyse by sequencing the surviving progeny, may be the ones that underpin the sex distortion observed, and may be subject to fundamentally different repair events.

A number of questions remain to be answered, such as the mechanism through which Xshredding causes the loss of X-bearing gametes and how insufficient or incomplete DNA repair is associated with this process. This outcome is by no means self-evident; for example, it has been shown that functional sperm can be produced despite lacking either one or both major autosomes or lacking DNA [30]. The fly model of X-shredding we have established will allow to tackle these questions experimentally. Much remains to be understood also before Xshredders could move towards application. For example, CRISPR-induced X chromosome rearrangements or pathways for the evolution of cleavage-resistant repeats are areas that have not been sufficiently explored.

We also established an X-poisoning system in the Drosophila model by targeting X-linked ribosomal genes and achieved high rates of male bias. This latter strategy, in the form of a Ylinked endonuclease, has recently been proposed as an efficient alternative to gene drives for genetic control [8]. Modelling suggests Y-linked editors would outperform other self-limiting strategies while having less impact on non-target populations when compared to gene drives or driving Y-chromosomes. We found the mechanism of embryo lethality in the case of the ribosomal RpS6 gene to be more complex than anticipated. Rather than protein dose insufficiency, a dominant lethal effect may partially or totally explain our results. Such an effect in mutated ribosomal proteins has been observed previously [31] and could, for example, be explained by the poisoning of ribosomes with dysfunctional ribosomal proteins. Since, in this case, every escaper female represents only a single repair event, phenotypes would be expected to vary depending on the outcome of DNA repair. Another possibility to explain the survival of females with frameshift mutations in the target gene is that the haplolethality of RpS6 could be time-dependent so that after passing a "critical" stage of development, e.g. embryo development, the gene becomes haplosufficient. Further modelling may be required to understand how these phenotypes would impact genetic control at the population level. 
The $\mathrm{X}$-shredding and the $\mathrm{X}$-poisoning transgenes we have described here could be expressed from the Drosophila Y chromosome which has recently been modified to harbour pre-characterized gRNA sites for transgene insertion [32]. This could allow, for the first time, to explore the use of such Y-linked editors with gRNAs for X-poisoning or X-shredding. To what degree these two strategies could be designed around or would be susceptible to X-chromosome inactivation in the male germline of Drosophila is a further research question, in particular as this process is not fully understood in the fly [33,34]. In both strategies timing of expression appears key and too early expression-when Cas9 was driven by the nos promoter -was unsuccessful, resulting in no distortion for the $\mathrm{X}$-shredding and male sterility for the $\mathrm{X}$ poisoning strategy.

Nevertheless, the application of X-poisoning to agriculturally or medically relevant species would appear to be a more straightforward proposition in particular as ribosomal target genes show high levels of conservation and because lower levels of Cas 9 activity for cleaving a single target site per chromosome may suffice. Also, the characterization of $Y$ chromosomes and the identification of male determining regions $[7,35,36]$ and docking sites [32] within which to land transgenes has recently made great progress.

\section{Materials and methods}

\section{Identification of candidate kmers for $\mathrm{X}$-shredding}

Error-corrected PacBio reads derived from males of the ISO1 strain (ref doi: 10.1038/nbt. 3238) were obtained from the University of Maryland Center for Bioinformatics and Computational Biology (http://gembox.cbcb.umd.edu/mhap/data/dmel.polished.fastq.gz). Illumina reads from males and females of the same strain were obtained from the NCBI short read archive (female: SRX826515 and male: SRX826516). These were used as inputs to run Redkmer [12]. The mitochondrial genome (NCBI accession number: NC_024511) was used to filter out mitochondrial derived reads. We only considered Pacbio reads between $2 \mathrm{~kb}$ and $100 \mathrm{~kb}$ to improve chromosome quotient accuracy (CQ-[37]) and excluded kmers occurring less than 4 times in the combined male and female Illumina data. We applied a number of critera to the 25,298 candidate X-kmers predicted by Redkmer to be X-linked abundant sequences. First, we used FlashFry [38] to select among the candidate X-kmers, those that contained a sequence suitable for the design of a Cas9 gRNA. To estimate off-target potential, we used as a reference all PacBio reads predicted by Redkmer to represent autosomal and $\mathrm{Y}$ derived sequences. We then applied several additional metrics such as the maximum number of occurrences of a kmer per PacBio read, giving an estimate of the repeat size, and the total number PacBio reads containing the kmer at least once, giving an estimate of the abundance of the repeat across the X-chromosome. Kmers shortlisted for testing were selected based on the following cutoffs: abundance in the genome (coverage in combined male and female Illumina data $>\log _{10}(2)$, coverage in PacBio data $>\log _{10}(1)$, the maximum kmer hits per PacBio read $>7.5$, and at least 5 perfect blast hits to the assembled genome. All candidate X-kmers were also re-assembled into longer contigs using the Geneious Software (Geneious Assembler allowing no mismatches) to identify possible higher-order repeat loci and to ensure that different sequence classes would be targeted in our experiments. A total of 8 of $205 \mathrm{X}$-kmers passing all filters were then selected for testing in transgenic strains.

\section{Generation of transgenic lines}

Design and assembly of constructs. Unless otherwise noted, cloning was performed with the NEBuilder Hi Fi DNA Assembly kit (New England Biolabs). PCR reactions were performed with the Phusion High-Fidelity PCR Master Mix with HF buffer (New England 
Biolabs). All inserts were verified by sequencing (GENEWIZ). Primers used for plasmid construction are listed in S9 Table.

Btub85D-cas9 expression plasmid. The previously described plasmid pYSC47 $w^{-}$harbouring the 3Px3-eGFP transformation marker, attB and piggyBac recombination sequences (a gift from Andrea Crisanti, Imperial College London) was used to build Drosophila cas 9 constructs. The plasmid was linearized with Sbfl. A 478 bp DNA fragment of $\beta t u b 85 D 5$ ' regulatory region was amplified with primers $\beta t u b 85 D$ F/ $/$ tub85D-cas $9-R$ from the genomic DNA of Drosophila strain $w^{1118}$. The human codon-optimized cas 9 coding sequence including two nuclear localization signals (SV40 NLS at the 5' and nucleoplasmin NLS at the 3') was amplified from hcas9 (a gift from George Church; Addgene plasmid \# 41815; http://n2t.net/addgene:41815; RRID: Addgene_41815) using primers $\beta t u b 85 D$-cas $9-F /$ cas $9-\beta t u b 56 D-3{ }^{\prime} U T R-R$ [39]. A third DNA fragment of $651 \mathrm{bp}$ in the $\beta$ tub56D 3'-UTR region was amplified with primers cas $\beta$ tub56F/ $\beta$ tub56D R from the plasmid $p$ YSC61. The three fragments were cloned in the open backbone of $S b f f$-linearized $p Y S C 47 w$ " resulting in the final plasmid termed " $p Y S C 47 w+\beta t u b 85 D_{-}$cas9_Btub56D". The plasmid was used for both piggyBac and attP-docking site integration.

Btub85D-Lbcpf1 expression plasmid. To generate the plasmid expressing Lbcpf1, fragments containing the $\beta t u b 85 D$ promoter and the $\beta t u b 56 D$ 3'-UTR were amplified from the construct $p Y S C 47 w+\beta t u b 85 D \_c a s 9 \_\beta t u b 56 D$. The fragment containing the $L b c p f 1$ coding sequence was amplified from $p Y 016$ hLbcpf1 (Addgene plasmid \# 69988; http://n2t.net/ addgene:69988; RRID:Addgene_69988) [40]. The three fragments were finally assembled in Sbfl-digested $p$ YSC47 $w^{-}$.

Guide RNA expression plasmid. All single guide RNAs were cloned in BbsI-linearized pCFD3-dU6:3 gRNA plasmid (a gift from Simon Bullock; Addgene plasmid \# 49410; http://n2t. net/addgene:49410; RRID:Addgene_49410) [17] and harbouring a U6 promoter. A gRNA array containing Muc14a_4, hydra_1, esi2.1_1 and $w \_e x 3 \_2$, in this order, was assembled with the oligos Array1_cas9_PCR1_F, Array1_cas9_PCR1_R, Array1_cas9_PCR2_F, Array1_ cas9_PCR2_R and Array1_cas9_PCR3_R and cloned in $p C F D 5$ plasmid (a gift from Simon Bullock; Addgene plasmid \# 73914; http://n2t.net/addgene:73914; RRID:Addgene_73914) [18]. Two distinct arrays, each containing two gRNAs targeting ribosomal genes, were assembled with primers $R p S 6$ 2_pCFD4_F and RpS6_ 1_pCFD4_R for the RpS6_2 + RpS6_1 array and RpS6_2_pCFD4_F and RpS5a_1_pCFD4_R for the RpS6_2 + RpS5a_1 array. The arrays were cloned in $p C F D 4-U 6: 1 \_U 6: 3$ tandem gRNAs plasmid (a gift from Simon Bullock; Addgene plasmid \# 49411; http://n2t.net/addgene:49411; RRID:Addgene_49411). The plasmids $p C F D 3, p C F D 4$ and $p C F D 5$ were all integrated in attP-docking sites. CRISPR target site design. CHOPCHOP v2 was used to choose gRNA target sites in RpS6 and RpS5a ribosomal genes specific regions in Drosophila genome (dm6).

Embryo injections. Embryo injections were carried out at the University of Cambridge Fly Injection Facility. The $\beta t u b 85 D \_c a s 9$ constructs were inserted at the $P\{C a r y P\} a t t P 40$ site on the 2nd chromosome (25C6; Stock 13-20) and the PBac\{ $\left.y^{+}-a t t P-9 A\right\} V K 00027$ on the 3rd chromosome (89E11; Stock 13-23), both stocks marked with yellow ${ }^{+}$. Moreover, the $\beta t u b 85 D \_c a s 9$ plasmid was integrated in D. melanogaster by piggyBac transposition. Transgenic flies were balanced with $w^{1118}$; if/CyO and $w^{1118}$; TM3, Sb/TM6B. Different lines were generated with $\beta t u b 85 D \_c a s 9$ integrated on the $\mathrm{X}(20 \mathrm{D})$, on the second (20G) and on the third chromosome (20F). gRNAs cloned in $p C F D 3, p C F D 4$ or $p C F D 5$ were integrated in the genome at the $P\{$ CaryP $\} a t t P 40$ site on the second (Bloomington stock 25709) and/or $P\{$ CaryP $\}$ attP2 site on the third chromosome (Bloomington stock 25710).pCDF4_RpS6_2_RpS6_1, pCDF4_RpS6_2_RpS5a_1, pCFD3_w_ex3-2, pCFD3_RpS6_gRNA2, pCFD3_RpS6_gRNA1, pCFD3_Muc14a_1,pCFD5_Cas9_Array1, pCFD3_CG33235_1,pCFD3_CG15040_1, pCFD3_Hydra_1,pCFD3_Muc14a_4,pCFD3_Muc14a_3,pCFD3_Muc14a_3,pCFD3_ 
Muc14a_5,pCFD3_Muc14a_6, and w_ex3-1 (LbCpf1) were inserted in the $P\{C a r y P\} a t t P 2$ site on the third chromosome and $p C F D 3 \_R p S 5 a \_g R N A 1, p C F D 3 \_R p S 5 a_{-} g R N A 2, p C F D 3 \_$ Muc14a_2, pCFD3_Hydra_1,pCFD3_Muc14a_3,pCFD3_Muc14a_5, and pCFD3_ $M u c 14 a \_6$ were inserted in the $P\{C a r y P\} a t t P 40$ site on the second chromosome.

\section{Fly husbandry and strains}

Flies were maintained under standard conditions at $25^{\circ} \mathrm{C}$ with a $12 / 12$ hour day and night cycle. For general maintenance, stocks were provided with new food every 2-3 weeks. Flies were anesthetized with $\mathrm{CO}_{2}$ during phenotyping. To assess fluorescent green eyes phenotype conferred by the 3PX3:::eGFP transgene, we used a conventional fluorescence microscope. Description of stocks not provided here can be found in FlyBase (http://flybase.org). The lines used in this study were: DNAlig $4^{57}$ Bloomington Stock \#8520, $S p n-A^{057}$ and $S p n-A^{093}$ a gift from Mitch McVey (Tuft University), Df(3R)XF3 Bloomington Stock \#2352, UAS- $\alpha$-spectrinRNAi Bloomington Stock \#56932 (TRIP.HMC04371), nanos_Gal4 Bloomington Stock \#4937, C (1)DX/FM6 (compound chromosome with attached X-chromosomes) Bloomington stock $\# 784$.

\section{Analysis of $\beta$ tub85D-cas9 and nos-cas9 activity}

To test attP or piggyBac integrated $\beta t u b 85 D$-cas 9 and nos-cas 9 efficiencies, females from each line were crossed to $w^{+} ; w_{-}$ex3-2 gRNA males bearing a gRNA targeting the white gene on the $\mathrm{X}$-chromosome. Red eye trans-heterozygous $w^{+}$; cas $9 / w \_e x 3-2 \mathrm{~F} 1$ males were selected, crossed to $w^{1118}$ females and the percentage of white eye females in the progeny was recorded.

\section{Genetic crosses for $\mathrm{X}$-shredding and $\mathrm{X}$-poisoning}

During the initial screen for $\mathrm{X}$-shredding-induced sex ratio distortion, $\beta$ tub85D-cas 9 mothers were crossed to each gRNA line (from a 25 nucleotides kmer), in a vial at $25^{\circ} \mathrm{C}$. At least twenty trans-heterozygous $\mathrm{F} 1 \mathrm{gRNA} /+; \beta t u b 85 D$-cas $9 /+$ males for each combination of sgRNA were then crossed to the same number of $w^{-}$females in new vials at the same temperature. We used the reverse crosses, $\beta$ tub85D-cas $9 / \mathrm{sgRNA}$ females crossed to $w^{-}$males, as controls due to the lack of $\beta t u b 85 D$ activity in females. We discarded the gRNA lines that did not result in sex ratio distortion while those where we recorded a male bias progeny were further tested by pursuing single cross analysis. For every single cross, a single trans-heterozygous $\beta$ tub85D-cas $9 /+$; $g R N A /+$ male (experiment) and a heterozygous $\beta t u b 85 D$-cas $9 /+$ male (control) were crossed to three wild-type females in separate vials at $25^{\circ} \mathrm{C}$. We set up a minimum of ten single crosses for each genotype analysed. For all crosses where we analysed the change in male bias in the progeny of $\beta t u b 85 D$-cas9/+; Muc14a_6/+ in a NHEJ, HDR or fusome mutant backgrounds, we set up ten-twenty replicas of single male crosses, i.e., $w$ DNAlig4 ${ }^{57}$; Muc14a_6/Btub85D-

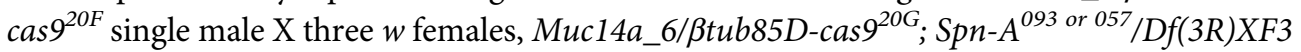
single male X three $w$ females and UAS- $\alpha$-spectrinRNAi/Muc14a_6; $\beta$ tub85D-cas $9^{20 F} /$ nanos_Gal4 single male X three $w$ females and compared the results to the progenies of Muc14a_6/ $\beta$ tub85D-cas 9 and $\beta$ tub85D-cas9/+ single male crosses used as controls.

In all crosses for $\mathrm{X}$-poisoning-induced sex ratio distortion, $\beta$ tub85D-cas 9 mothers were crossed to each gRNA analysed, in a vial. Single F1 $\beta t u b 85 D-\operatorname{cas} 9 /+; g R N A /+$ (experiment) and $\beta$ tub85D-cas9/+(control) males were then crossed to three wild-type females in separate vials. We set up a minimum of ten single crosses for each genotype to generate means and standard deviations for statistical comparisons and thus measure consistency and robustness of the results. All crosses were done at $25^{\circ} \mathrm{C}$. Percent of males and females was calculated as the ratio between the number of individuals counted and the number of individuals expected for each 
genotype. Flies were scored and examined with the Nikon SMZ1500 stereomicroscope equipped with a CoolLED $p$ E-300 Led fluorescent illumination.

\section{In vitro cleavage assay}

The Muc14a_3, Muc14a_4, Muc14a_5 and Muc14a_6 gRNAs were tested for in vitro activity using the Guide-it sgRNA In Vitro Transcription and Screening System (Takara Bio USA, Inc.). Each gRNA was transcribed and purified according to the In Vitro Transcription of sgRNA protocol. Two experiments were performed, allowing transcription to take place for 4 and 8 hours. Genomic DNA templates were obtained from Muc14a fragments previously cloned in a pMiniT 2.0 vector using PCR Cloning Kit (NEB) and primers kmer Muc14_F and 58537687_F. The genomic target was amplified by PCR with Cloning Analysis Forward and Reverse Primers, mapping in the pMiniT 2.0 plasmid. The amplicon corresponds to a genomic fragment of $1395 \mathrm{bp}$ and sequencing confirmed the presence of target sites for all 4 gRNAs. For in-vitro digestions the DNA was excised from an agarose gel and purified with Monarch DNA Gel Extraction Kit (NEB). DNA concentration and absorbance ratio were measured with a NanoDrop Spectrophotometer (ThermoScientific). PCR amplification of target DNA and a Cas9 cleavage assay were then carried out according to the protocol.

\section{Amplicon sequencing analysis preparation}

Genomic DNA was isolated from ten single F1 males originated from the cross $\beta t u b 85 D_{-}$cas $9^{20 F} / M u c 14 a \_6 \mathrm{X} C(1) D X / F M 6$ (compound chromosome with attached X-chromosomes) Bloomington stock \#784 and from one control male from the cross $w / Y$; $\beta t u b 85 D \_c a s 9^{20 F}$ / TM6B using the QIAamp DNA Micro Kit. Genomic loci containing the Muc14a_6 gRNA target site were amplified with Phusion HF DNA polymerase (Thermo Scientific) using Repeat 56910823_illumina_F and Repeat_Muc14a_3_illumina_R primers containing the Illumina adapters. $200 \mathrm{ng}$ of genomic DNA in a $100 \mu \mathrm{l}$ reaction volume were used as a template for a limiting PCR reaction to amplify 153 bp of slightly different repeats in the Muc14a gene. To maintain the proportion of reads corresponding to particular repeats, the PCR reactions were performed under non-saturating conditions for a total of 25 cycles with $55^{\circ} \mathrm{C}$ annealing temperature.

For deep sequencing analysis of the ribosomal gene $R p S 6$ target site, the genomic DNA was extracted from a pool of four daughters from the cross $\beta t u b 85 D \_c a s 9^{20 F} / R p S 6 \_2 \mathrm{X} w$, four daughters from the cross $\beta t u b 85 D_{-} c a s 9^{20 F} / R p S 6 \_2+R p S 5 a \_1 \mathrm{X} w$ and from the $\beta t u b 85 D_{-}-$ cas $9^{20 F} /+$ daughter control. The primers RpS6_1F and RpS6_1R were used to amplify a $234 \mathrm{bp}$ DNA fragment spanning the RpS6_2 target site. The amplicons were purified with NEB Monarch PCR \& DNA Cleanup Kit and quantified with Nanodrop. $200 \mathrm{ng}$ were checked on a gel and $500 \mathrm{ng}$ were sent to GENEWIZ to be sequenced with NGS-based amplicon sequencing. We ran CRISPResso [41] software on raw sequencing data to detect mutations at the target site using parameter -q 30 , setting the minimum average read quality score (phred33) to 30 .

\section{Viability studies}

To identify the developmental stage at which the progeny from $M u c 14 a \_6 / \beta t u b 85 D \_c a s 9$ and RpS6_2/Btub85D_cas 9 crossed to $w^{1118}$ die, we quantified egg hatching, pupae and adult death rates in the F1. To quantify the egg hatching rate, 20-30 heterozygous Muc14a_6/קtub85D_cas 9 or RpS6_2/ $/$ tub $85 D \_c a s 9$ and $20-30 w^{1118}$ virgin females were set up in embryo collection cages with grape juice agar plates and yeast paste. Two different embryo collection cages with $w^{1118}$ and $\beta$ tub85D_cas9/+ males crossed to $w^{1118}$ females served as a comparison control. Between four and six collections of 70-200 embryos each, were performed for each genotype. 
Every embryo collection was transferred in a separate fly vial and followed for over $36 \mathrm{~h}$ to count the number of embryos that did not hatch, the number of pupae, and female and male adults. Percent survival to each stage was calculated as the ratio between the number of individuals counted and the number of individuals expected for each genotype. The data for the two experiments and for each of the crosses are shown in Table Fig 2C and Table Fig 4C in supplementary Dataset 1 .

\section{Supporting information}

S1 Fig. Evaluation of Cas9 activity when expressed from $\beta t u b 85 D$-cas9 and $\beta t u b 85 D \_c p f 1$ transgenes integrated at various genomic locations (chromosome indicated in parentheses) using $\phi \mathrm{C} 31$ (attP) or piggyBac mediated transformation. Flies carrying $\beta t u b 85 D$-cas 9 expressed from attP docking \#15-1 on the second and \#10-1 and \#4 on the third chromosomes and from piggyBac mediated random integrations \#20D on the $\mathrm{X}, \# 20 \mathrm{G}$ on the second and $\# 20 \mathrm{~F}$ and $\# 20 \mathrm{C}$ on the third chromosomes were crossed to lines transgenic for $w \_e x 3-2$ gRNA that targets the white gene on the X-chromosome. $\beta t u b 85 D$-cas9/w_ex3-2 F1 males with red eyes were then crossed to white mutant females, and the female progeny scored for white eyes. Experiments combining two $\beta t u b 85 D$-cas 9 transgenes with the $w \_$ex3-2 gRNA and $\beta$ tub85D-cas 9 with $w_{-}$ex3-2 expressed as part of a gRNA multiplex array were also performed. Similarly, $\beta$ tub85D_cpf1/w_ex3-1 red eye males were crossed to white females, and the female progeny scored for white eyes. Along with the $\beta t u b 85 D$ promoter, the nanos-cas 9 transgene efficiency was tested with the same $w_{-}$ex3-2 gRNA. gRNAs used for each experiment are shown below the graph. $\mathrm{n}$ is the number of individuals (males + females) in the F1 progeny. (TIF)

S2 Fig. Candidate $\mathrm{X}-\mathrm{kmer}$ abundance and chromosomal distribution. (A) Coverage of all candidate X-kmers (grey dots) including those X-kmers chosen for experimental evaluation (colored dots) within the Illumina and PacBio whole-genome sequencing read datasets as predicted by Redkmer. (B) Genomic distribution of all candidate X-kmers (grey dots) and those selected X-kmers for experimental testing (colored dots) on D. melanogaster chromosome arms based on perfect complementarity. The $\mathrm{X}$-axis indicates the Mbp position of matches on each chromosomal arm, including the $4^{\text {th }}$ chromosome, unmapped contigs and heterochromatin $\left(\_h\right)$.

(TIF)

S3 Fig. Kmer sub-selection applied to Redkmer output. Criteria for candidate kmer selection (X-axes) are shown for each of the eight selected X-kmers for X-shredding (colored dots) and for the remaining candidate X-kmers (grey dots). Red vertical lines highlight the minimum cutoff values imposed for the final target site selection. Density plots of each criteria are also shown for the entire Redkmer candidate X-kmer output. The part of the kmer sequence that represents the target sites of experimental gRNAs is indicated (underline).

S1 Table. Reported in the table are the numbers and percentages of female progenies from different genetic crosses using one or two cas 9 or the $c p f 1$ endonucleases driven by either the $\beta t u b 85 D$ or the nanos promoters, to assess the efficiency of cleavage in the white gene. All crosses are between males bearing the endonuclease and the sgRNA, $w \_$ex3-2 and $w \_e x 3-1$ for cas 9 and cpf1 respectively, and females of the white genotype. Different $\beta t u b 85 D$-cas 9 lines, generated by attP or piggyBac integrations, are compared. The Array1_1 is a multiplex of 4 different gRNAs (Muc14a_4, hydra_1, esi-2.1_1 and w_ex3-2).

(XLSX) 
S2 Table. Predicted perfect blastn hits of kmer target sequences for X-shredding within the Drosophila melanogaster heterochromatin-enriched genome assembly [13]. (XLSX)

S3 Table. Reported in the table are the numbers and percentages of male and female progenies from genetic crosses between of $\beta$ tub85D-cas9/gRNA and wild-type $w$ females to assess the sex-ratio. sgRNA lines are complementary to single (Muc14a_1, Muc14a_2, RpS6_1, RpS6_2, RpS5a_1 and RpS5a_2) or multiple hits on the X chromosome. Progeny of $\beta t u b 85 D$ cas 9/+ males crossed to wild type females (no gRNA) or from the reverse cross ( $\beta$ tub85D-cas9/ $g R N A$ females crossed to wild type males $=$ female control) were used as control. Crosses were set as pools of males and females or as multiple male single crosses for a minimum of ten independent single crosses.

(XLSX)

S4 Table. Analysis of combinations of gRNAs and Cas9 sources for X-shredding. Reported in the table are the numbers and percentages of males and females in the progeny from genetic crosses between of $\beta$ tub85D-cas9/gRNA and wild-type $w$ females. Average frequencies (\%) and standard deviations (STDEV) are shown for each parental genotype with a single copy of $\beta$ tub85D-cas 9 transgene (1X), two copies of $\beta$ tub85D-cas 9 transgene (2X) or one copy of nos-cas 9 (grey bars). All lines were crossed to wild type $w$ individuals. The reciprocal cross (female ctrl) or heterozygote $\beta$ tub85D-cas $9 /+$ or nos-cas $9 /+$ without gRNA (no gRNA) were used as control. Crosses were set as pools of males and females or as multiple male single crosses for a minimum of ten independent crosses. (XLSX)

S5 Table. Survival analysis of the F1 progeny of Muc14a_6/קtub85D-cas9 males crossed to $w$ females compared to $w$ and $\beta$ tub85D-cas9/+ control males crossed to $w$ females. F1 embryos were collected six, five and four times from cages containing males of the genotype Muc14a_6/קtub85D-cas $9,+/ \beta t u b 85 D$-cas 9 or white respectively, crossed to white females. The table shows the numbers of embryos, hatched embryos, pupae, females, males and total adults for each collection. The table also shows the percentages of pupae on collected embryos, adults on pupae, and frequencies of males and females on adults.

(XLSX)

S6 Table. Influence of DNA repair and fusome integrity on $\mathrm{X}$-shredding. The table shows male and female numbers, frequencies (\%) and standard deviation (STDEV) in the F1 of Muc14a_6/3tub85D-cas9 males with DNAlig4 ${ }^{57}$, spn- $A^{057}$ or $s p n-A^{093}$ mutations crossed to white females. Also shown are the F1 female and male frequencies for the cross of UAS_ $\alpha$-spectrinRNAi; nos-GAL4 (yellow) knockdown mutants. At least ten independent single crosses per experiment were performed and each cross was between one male and three females. (XLSX)

S7 Table. Efficiency of single gRNAs or combinations of gRNAs for X-poisoning. The table shows the numbers of males, females and percentage of males in the progeny from $\beta t u b 85 D$ cas 9 males combined with $g R N A$ lines and crossed to wild type white females. Individual gRNAs, RpS5a_1 or RpS5a_2, and RpS6_1 or RpS6_2 or double gRNA arrays co-expressing $R p S 6 \_2+R p S 5 a \_1$ or $R p S 6 \_2+R p S 6 \_1$ as well as a combination of both $R p S 6 \_2$ and $M u c 14 a \_6$ transgenes are shown. Control experiments consist of $\beta t u b 85 D$-cas9/+ (no gRNA) males crossed to white females. The table also shows the results from the cross between nos-cas9/RpS6_2 males and white females. The last two columns in the table show the average 
number of F1 individuals per single cross and the fraction of experimental versus control (set as 1 for control) averages. Each cross was between one male and three females and at least ten independent single crosses per experiment were performed.

(XLSX)

S8 Table. Survival analysis of the F1 progeny of RpS6_2/Btub85D-cas9 males crossed to $w$ females compared to $w$ and $\beta$ tub85D-cas9/+ control males crossed to $w$ females. F1 embryos were collected six, and five times from cages containing males of the genotype

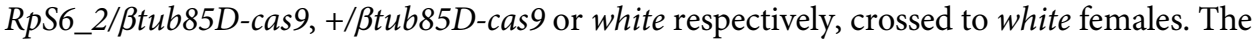
table shows the numbers of embryos, hatched embryos, pupae, females, males and total adults for each collection. The table also shows the percentages of pupae on collected embryos, adults on pupae, and frequencies of males and females on adults.

(XLSX)

S9 Table. Primers used in this study. The table shows the primers name, description of where they were used and the corresponding nucleotide sequences. The gRNA sequences are underlined.

(XLSX)

\section{Acknowledgments}

The authors would like to thank Steve Russell and Adam Phillippy for helpful discussions and for providing error-corrected whole genome sequence data. We would like to thank Alex Nash, Paolo Capriotti and Rita Colonna.

\section{Author Contributions}

Conceptualization: Barbara Fasulo, Philippos Aris Papathanos, Nikolai Windbichler.

Data curation: Barbara Fasulo, Angela Meccariello, Maya Morgan, Carl Borufka.

Formal analysis: Barbara Fasulo, Philippos Aris Papathanos.

Funding acquisition: Nikolai Windbichler.

Investigation: Nikolai Windbichler.

Methodology: Barbara Fasulo.

Project administration: Nikolai Windbichler.

Resources: Angela Meccariello.

Supervision: Nikolai Windbichler.

Validation: Barbara Fasulo, Philippos Aris Papathanos.

Visualization: Barbara Fasulo, Philippos Aris Papathanos.

Writing - original draft: Nikolai Windbichler.

Writing - review \& editing: Barbara Fasulo, Philippos Aris Papathanos, Nikolai Windbichler.

\section{References}

1. Hamilton WD. Extraordinary sex ratios. A sex-ratio theory for sex linkage and inbreeding has new implications in cytogenetics and entomology. Science. 1967; 156(3774):477-88. Epub 1967/04/28. https:// doi.org/10.1126/science.156.3774.477 PMID: 6021675. 
2. Sweeny TL, Barr AR. Sex Ratio Distortion Caused by Meiotic Drive in a Mosquito, Culex pipiens L. Genetics. 1978; 88(3):427-46. PMID: 17248804; PubMed Central PMCID: PMC1224591.

3. Newton M.E. WRLaSDI. A cytogenetic analysis of meiotic drive in the mosquito Aedes aegypti. Genetica. 1976; 46:297-318.

4. Windbichler N, Papathanos PA, Crisanti A. Targeting the $X$ chromosome during spermatogenesis induces $\mathrm{Y}$ chromosome transmission ratio distortion and early dominant embryo lethality in Anopheles gambiae. PLoS Genet. 2008; 4(12):e1000291. Epub 2008/12/06. https://doi.org/10.1371/journal.pgen. 1000291 PMID: 19057670; PubMed Central PMCID: PMC2585807.

5. Galizi R, Doyle LA, Menichelli M, Bernardini F, Deredec A, Burt A, et al. A synthetic sex ratio distortion system for the control of the human malaria mosquito. Nat Commun. 2014; 5:3977. Epub 2014/06/10. https://doi.org/10.1038/ncomms4977 PMID: 24915045; PubMed Central PMCID: PMC4057611.

6. Galizi R, Hammond A, Kyrou K, Taxiarchi C, Bernardini F, O'Loughlin SM, et al. A CRISPR-Cas9 sexratio distortion system for genetic control. Sci Rep. 2016; 6:31139. Epub 2016/08/03. https://doi.org/10. 1038/srep31139 PMID: 27484623; PubMed Central PMCID: PMC4971495.

7. Hall AB, Basu S, Jiang X, Qi Y, Timoshevskiy VA, Biedler JK, et al. SEX DETERMINATION. A maledetermining factor in the mosquito Aedes aegypti. Science. 2015; 348(6240):1268-70. Epub 2015/05/ 23. https://doi.org/10.1126/science.aaa2850 PMID: 25999371; PubMed Central PMCID: PMC5026532.

8. Burt A, Deredec A. Self-limiting population genetic control with sex-linked genome editors. Proc Biol Sci. 2018; 285(1883). Epub 2018/07/25. https://doi.org/10.1098/rspb.2018.0776 PMID: 30051868; PubMed Central PMCID: PMC6083257.

9. Bernardini F, Galizi R, Menichelli M, Papathanos P-A, Dritsou V, Marois E, et al. Site-specific genetic engineering of the Anopheles gambiae $Y$ chromosome. Proceedings of the National Academy of Sciences. 2014; 111(21):7600-5. https://doi.org/10.1073/pnas.1404996111 PMID: 24821795; PubMed Central PMCID: PMC4040617.

10. White-Cooper $\mathrm{H}$. Tissue, cell type and stage-specific ectopic gene expression and RNAi induction in the Drosophila testis. Spermatogenesis. 2012; 2(1):11-22. https://doi.org/10.4161/spmg.19088 PMID: 22553486

11. Kondo S, Ueda R. Highly improved gene targeting by germline-specific Cas 9 expression in Drosophila. Genetics. 2013; 195(3):715-21. Epub 2013/09/03. https://doi.org/10.1534/genetics.113.156737 PMID: 24002648; PubMed Central PMCID: PMC3813859.

12. Papathanos PA, Windbichler N. Redkmer: An Assembly-Free Pipeline for the Identification of Abundant and Specific X-Chromosome Target Sequences for X-Shredding by CRISPR Endonucleases. CRISPR J. 2018; 1(1):88-98. https://doi.org/10.1089/crispr.2017.0012 PMID: 30627701; PubMed Central PMCID: PMC6319322.

13. Chang $\mathrm{CH}$, Larracuente $\mathrm{AM}$. Heterochromatin-Enriched Assemblies Reveal the Sequence and Organization of the. Genetics. 2019; 211(1):333-48. Epub 2018/11/12. https://doi.org/10.1534/genetics.118. 301765 PMID: 30420487; PubMed Central PMCID: PMC6325706.

14. Marygold SJ, Roote J, Reuter G, Lambertsson A, Ashburner M, Millburn GH, et al. The ribosomal protein genes and Minute loci of Drosophila melanogaster. Genome Biol. 2007; 8(10):R216. https://doi.org/ 10.1186/gb-2007-8-10-r216 PMID: 17927810; PubMed Central PMCID: PMC2246290.

15. McKim KS, Dahmus JB, Hawley RS. Cloning of the Drosophila melanogaster meiotic recombination gene mei-218: a genetic and molecular analysis of interval 15E. Genetics. 1996; 144(1):215-28. PMID: 8878687; PubMed Central PMCID: PMC1207495.

16. Stewart MJ, Denell R. The Drosophila ribosomal protein S6 gene includes a 3' triplication that arose by unequal crossing-over. Mol Biol Evol. 1993; 10(5):1041-7. https://doi.org/10.1093/oxfordjournals. molbev.a040053 PMID: 8412647.

17. Port F, Chen HM, Lee T, Bullock SL. Optimized CRISPR/Cas tools for efficient germline and somatic genome engineering in Drosophila. Proc Natl Acad Sci U S A. 2014; 111(29):E2967-76. Epub 2014/07/ 09. https://doi.org/10.1073/pnas.1405500111 PMID: 25002478; PubMed Central PMCID: PMC4115528.

18. Port F, Bullock SL. Augmenting CRISPR applications in Drosophila with tRNA-flanked sgRNAs. Nat Methods. 2016; 13(10):852-4. Epub 2016/09/07. https://doi.org/10.1038/nmeth.3972 PMID: 27595403; PubMed Central PMCID: PMC5215823.

19. Romeijn RJ, Gorski MM, van Schie MA, Noordermeer JN, Mullenders LH, Ferro W, et al. Lig4 and rad54 are required for repair of DNA double-strand breaks induced by P-element excision in Drosophila. Genetics. 2005; 169(2):795-806. Epub 2004/11/15. https://doi.org/10.1534/genetics.104.033464 PMID: 15545651; PubMed Central PMCID: PMC1449100. 
20. Staeva-Vieira E, Yoo S, Lehmann R. An essential role of DmRad51/SpnA in DNA repair and meiotic checkpoint control. EMBO J. 2003; 22(21):5863-74. https://doi.org/10.1093/emboj/cdg564 PMID: 14592983; PubMed Central PMCID: PMC275421.

21. Lu KL, Yamashita YM. Germ cell connectivity enhances cell death in response to DNA damage in the. Elife. 2017; 6. Epub 2017/08/15. https://doi.org/10.7554/eLife.27960 PMID: 28809158; PubMed Central PMCID: PMC5577909.

22. Kaufman TC. A Short History and Description of. Genetics. 2017; 206(2):665-89. https://doi.org/10. 1534/genetics.117.199950 PMID: 28592503; PubMed Central PMCID: PMC5499179.

23. Smits AH, Ziebell F, Joberty G, Zinn N, Mueller WF, Clauder-Münster S, et al. Biological plasticity rescues target activity in CRISPR knock outs. Nat Methods. 2019; 16(11):1087-93. Epub 2019/10/28. https://doi.org/10.1038/s41592-019-0614-5 PMID: 31659326.

24. Dilthey AT, Jain C, Koren S, Phillippy AM. Strain-level metagenomic assignment and compositional estimation for long reads with MetaMaps. Nat Commun. 2019; 10(1):3066. Epub 2019/07/11. https:// doi.org/10.1038/s41467-019-10934-2 PMID: 31296857; PubMed Central PMCID: PMC6624308.

25. Menon DU, Coarfa C, Xiao W, Gunaratne PH, Meller VH. siRNAs from an X-linked satellite repeat promote X-chromosome recognition in Drosophila melanogaster. Proc Natl Acad Sci U S A. 2014; 111 (46):16460-5. Epub 2014/11/03. https://doi.org/10.1073/pnas.1410534111 PMID: 25368194; PubMed Central PMCID: PMC4246271.

26. Kim M, Ekhteraei-Tousi S, Lewerentz J, Larsson J. The X-linked 1.688 Satellite in. Genetics. 2018; 208 (2):623-32. Epub 2017/12/13. https://doi.org/10.1534/genetics.117.300581 PMID: 29242291; PubMed Central PMCID: PMC5788526.

27. Kuhn GC, Küttler H, Moreira-Filho O, Heslop-Harrison JS. The 1.688 repetitive DNA of Drosophila: concerted evolution at different genomic scales and association with genes. Mol Biol Evol. 2012; 29(1):711. Epub 2011/06/28. https://doi.org/10.1093/molbev/msr173 PMID: 21712468.

28. Champer SE, Oh SY, Liu C, Wen Z, Clark AG, Messer PW, et al. Computational and experimental performance of CRISPR homing gene drive strategies with multiplexed gRNAs. bioRxiv. 2019:679902. https://doi.org/10.1101/679902

29. Chan YS, Naujoks DA, Huen DS, Russell S. Insect population control by homing endonuclease-based gene drive: an evaluation in Drosophila melanogaster. Genetics. 2011; 188(1):33-44. https://doi.org/ 10.1534/genetics.111.127506 PMID: 21368273; PubMed Central PMCID: PMC3120159.

30. Lindsley DL, Grell EH. Spermiogenesis without chromosomes in Drosophila melanogaster. Genetics. 1969; 61(1):Suppl:69-78. Epub 1969/01/01. PMID: 5345403.

31. Devlin EE, Dacosta L, Mohandas N, Elliott G, Bodine DM. A transgenic mouse model demonstrates a dominant negative effect of a point mutation in the RPS19 gene associated with Diamond-Blackfan anemia. Blood. 2010; 116(15):2826-35. Epub 2010/07/06. https://doi.org/10.1182/blood-2010-03-275776 PMID: 20606162; PubMed Central PMCID: PMC2974590.

32. Buchman A, Akbari OS. Site-specific transgenesis of the Drosophila melanogaster Y-chromosome using CRISPR/Cas9. Insect Mol Biol. 2019; 28(1):65-73. Epub 2018/10/08. https://doi.org/10.1111/ imb.12528 PMID: 30079589.

33. Vibranovski MD, Zhang YE, Kemkemer C, Lopes HF, Karr TL, Long M. Re-analysis of the larval testis data on meiotic sex chromosome inactivation revealed evidence for tissue-specific gene expression related to the drosophila X chromosome. BMC Biol. 2012; 10:49; author reply 50. Epub 2012/06/12. https://doi.org/10.1186/1741-7007-10-49 PMID: 22691264; PubMed Central PMCID: PMC3391172.

34. Mikhaylova LM, Nurminsky DI. Lack of global meiotic sex chromosome inactivation, and paucity of tissue-specific gene expression on the Drosophila X chromosome. BMC Biol. 2011; 9:29. Epub 2011/05/ 04. https://doi.org/10.1186/1741-7007-9-29 PMID: 21542906; PubMed Central PMCID: PMC3104377.

35. Meccariello A, Salvemini M, Primo $P$, Hall B, Koskinioti $P$, Dalikova M, et al. Maleness-on-the-Y (MoY) orchestrates male sex determination in major agricultural fruit fly pests. Science. 2019; 365 (6460):1457-60. Epub 2019/08/31. https://doi.org/10.1126/science.aax1318 PMID: 31467189.

36. Krzywinska E, Dennison NJ, Lycett GJ, Krzywinski J. A maleness gene in the malaria mosquito Anopheles gambiae. Science. 2016; 353(6294):67-9. https://doi.org/10.1126/science.aaf5605 PMID: 27365445.

37. Hall AB, Papathanos PA, Sharma A, Cheng C, Akbari OS, Assour L, et al. Radical remodeling of the $Y$ chromosome in a recent radiation of malaria mosquitoes. Proc Natl Acad Sci U S A. 2016; 113(15): E2114-23. Epub 2016/03/29. https://doi.org/10.1073/pnas.1525164113 PMID: 27035980; PubMed Central PMCID: PMC4839409.

38. McKenna A, Shendure J. FlashFry: a fast and flexible tool for large-scale CRISPR target design. BMC Biol. 2018; 16(1):74. Epub 2018/07/05. https://doi.org/10.1186/s12915-018-0545-0 PMID: 29976198; PubMed Central PMCID: PMC6033233. 
39. Mali P, Yang L, Esvelt KM, Aach J, Guell M, DiCarlo JE, et al. RNA-guided human genome engineering via Cas9. Science. 2013; 339(6121):823-6. Epub 2013/01/05. https://doi.org/10.1126/science.1232033 PMID: 23287722; PubMed Central PMCID: PMC3712628.

40. Zetsche B, Gootenberg JS, Abudayyeh OO, Slaymaker IM, Makarova KS, Essletzbichler P, et al. Cpf1 is a single RNA-guided endonuclease of a class 2 CRISPR-Cas system. Cell. 2015; 163(3):759-71. Epub 2015/10/01. https://doi.org/10.1016/j.cell.2015.09.038 PMID: 26422227; PubMed Central PMCID: PMC4638220.

41. Pinello L, Canver MC, Hoban MD, Orkin SH, Kohn DB, Bauer DE, et al. Analyzing CRISPR genomeediting experiments with CRISPResso. Nat Biotechnol. 2016; 34(7):695-7. Epub 2016/07/13. https:// doi.org/10.1038/nbt.3583 PMID: 27404874; PubMed Central PMCID: PMC5242601. 